\title{
Herschel-ATLAS: revealing dust build-up and decline across gas, dust and stellar mass selected samples - I. Scaling relations
}

\author{
P. De Vis, ${ }^{1,2 \star}$ L. Dunne, ${ }^{3,4}$ S. Maddox,${ }^{3,4}$ H. L. Gomez, ${ }^{3}$ C. J. R. Clark, ${ }^{3}$ A. E. Bauer, ${ }^{5}$ \\ S. Viaene, ${ }^{2}$ S. P. Schofield, ${ }^{3}$ M. Baes,${ }^{2}$ A. J. Baker, ${ }^{6}$ N. Bourne, ${ }^{3}$ S. P. Driver,${ }^{7}$ S. Dye, ${ }^{8}$ \\ S. A. Eales, ${ }^{2}$ C. Furlanetto, ${ }^{8,9}$ R. J. Ivison, ${ }^{4,10}$ A. S. G. Robotham, ${ }^{7}$ K. Rowlands,${ }^{11}$ \\ D. J. B. Smith, ${ }^{12}$ M. W. L. Smith, ${ }^{2}$ E. Valiante ${ }^{2}$ and A. H. Wright ${ }^{7}$ \\ ${ }^{1}$ Department of Physics \& Astronomy, University of Canterbury, Private Bag 4800, Christchurch 8140, New Zealand \\ ${ }^{2}$ Sterrenkundig Observatorium, Universiteit Gent, Krijgslaan 281, B-9000 Gent, Belgium \\ ${ }^{3}$ School of Physics \& Astronomy, Cardiff University, Queen's Buildings, The Parade, Cardiff CF24 3AA, UK \\ ${ }^{4}$ Institute for Astronomy, The University of Edinburgh, Royal Observatory, Blackford Hill, Edinburgh EH9 3 HJ, UK \\ ${ }^{5}$ Australian Astronomical Observatory, PO Box 915, North Ryde, NSW 1670, Australia \\ ${ }^{6}$ Department of Physics and Astronomy, Rutgers, The State University of New Jersey, 136 Frelinghuysen Road, Piscataway, NJ 08854-8019, USA \\ ${ }^{7}$ International Centre for Radio Astronomy Research, The University of Western Australia, Crawley, Perth 6009, Australia \\ ${ }^{8}$ School of Physics \& Astronomy, University of Nottingham, University Park, Nottingham NG7 2RD, UK \\ ${ }^{9}$ CAPES Foundation, Ministry of Education of Brazil, Braslia/DF 70040-020, Brazil \\ ${ }^{10}$ European Southern Observatory, Karl Schwarzschild Strasse 2, Garching D-85748, Germany \\ ${ }^{11}$ School of Physics \& Astronomy, University of St Andrews, North Haugh, St Andrews KY16 9SS, UK \\ ${ }^{12}$ Centre for Astrophysics, Science \& Technology Research Institute, University of Hertfordshire, Hatfield, Herts AL10 9AB, UK
}

Accepted 2016 September 29. Received 2016 September 14; in original form 2015 December 25

\begin{abstract}
We present a study of the dust, stars and atomic gas $\left(\mathrm{H}_{\mathrm{I}}\right)$ in an $\mathrm{H}_{\mathrm{I}}$-selected sample of local galaxies $(z<0.035)$ in the Herschel Astrophysical Terahertz Large Area Survey fields. This $\mathrm{H}$ I-selected sample reveals a population of very high gas fraction ( $>80$ per cent), low stellar mass sources that appear to be in the earliest stages of their evolution. We compare this sample with dust- and stellar-mass-selected samples to study the dust and gas scaling relations over a wide range of gas fractions (proxy for evolutionary state of a galaxy). The most robust scaling relations for gas and dust are those linked to near-ultraviolet $-r$ (specific star formation rate) and gas fraction; these do not depend on sample selection or environment. At the highest gas fractions, our additional sample shows that the dust content is well below expectations from extrapolating scaling relations for more evolved sources, and dust is not a good tracer of the gas content. The specific dust mass for local galaxies peaks at a gas fraction of $\sim 75$ per cent. The atomic gas depletion time is also longer for high gas fraction galaxies, opposite to the trend found for molecular gas depletion time-scale. We link this trend to the changing efficiency of conversion of $\mathrm{H}_{\mathrm{I}}$ to $\mathrm{H}_{2}$ as galaxies increase in stellar mass surface density during their evolution. Finally, we show that galaxies start out barely obscured and increase in obscuration as they evolve, yet there is no clear and simple link between obscuration and global galaxy properties.
\end{abstract}

Key words: dust, extinction-ISM: evolution-galaxies: dwarf-galaxies: evolutiongalaxies: fundamental parameters - galaxies: ISM.

\section{INTRODUCTION}

About 30-50 per cent of the optical/ultraviolet (UV) radiative energy produced by stars and active galactic nuclei (AGNs) in galaxies is absorbed by dust and thermally re-emitted in the far-infrared

^E-mail: pieter.devis@pg.canterbury.ac.nz
(FIR) and submillimetre (submm) parts of the spectrum (Fixsen et al. 1996; Driver et al. 2016; Viaene et al. 2016). It is therefore difficult to develop a thorough understanding of galaxy evolution without also understanding the interstellar medium (ISM). Dust properties have been investigated for several decades using space missions such as IRAS (Neugebauer et al. 1984), ISO (Kessler et al. 1996) and Spitzer (Werner et al. 2004), and ground-based submm instruments such as Submillimetre Common-User Bolometer Array 
(Holland et al. 1999), MAMBO (Kreysa et al. 1998) and LABOCA (Kreysa et al. 2003). However, with the advent of the Herschel Space Observatory ${ }^{1}$ (Pilbratt et al. 2010) we have entered a new era for interstellar dust studies. Herschel has superior angular resolution and sensitivity compared to previous FIR facilities and operates right across the peak of the dust spectral energy distribution (SED; $70-500 \mu \mathrm{m})$. This makes it sensitive to the diffuse cold $(T<25 \mathrm{~K})$ dust component that dominates the dust mass in galaxies (Devereux \& Young 1990; Dunne \& Eales 2001; Draine et al. 2007; Clark et al. 2015), as well as warmer $(T>30 \mathrm{~K})$ dust radiating at shorter wavelengths which often dominates the dust luminosity. The consensus is that the warm dust component is heated by star-forming regions (Devereux \& Young 1990; Kennicutt 1998; Calzetti et al. 2007; Boquien et al. 2010; Verley et al. 2010; Bendo et al. 2012), and the cold dust component (which makes up the bulk of the dust mass) can be heated by both star-forming regions and older stellar populations (Bendo et al. 2015).

Herschel is uniquely suited to studying the role played by dust in the evolutionary history of galaxies. The first logical step is to quantify how the dust content of galaxies varies with galactic properties such as stellar mass, colour, gas content, star formation rate (SFR) and other parameters. The resulting scaling relations provide vital information about the interplay of dust, gas and the star formation cycle, leading to important insights into the physical processes regulating galaxy evolution (e.g. Dunne et al. 2011) and providing strong constraints on chemical evolution models (e.g. Rowlands et al. 2014b; Zhukovska 2014). Before Herschel, the main scaling relations studied were global relations between dust, gas and stellar masses (e.g. Devereux \& Young 1990; Sanders, Scoville \& Soifer 1991; Dunne et al. 2000; Driver et al. 2007) and the evolution of the dust-to-gas ratio with stellar mass and metallicity (e.g. Issa, MacLaren \& Wolfendale 1990; Lisenfeld \& Ferrara 1998; James et al. 2002; Draine et al. 2007). These studies showed a strong correlation between dust and gas masses, and found an increase of the dust-to-gas mass ratio as a function of stellar mass and metallicity, though there is often disagreement in the exact slope of the relationships. More recently, da Cunha et al. (2010) used IRAS data to show that the dust-to-stellar mass ratio strongly correlates with specific star formation rate (SSFR), as predicted by chemical evolution models. This result has since been supported by further Herschel studies (Smith et al. 2012; Sandstrom et al. 2013; Rowlands et al. 2014a).

Since then, Herschel has expanded on these studies to include the cold dust component and explored a much wider range of galaxy types and luminosities, in far greater numbers, than was possible previously. The Herschel Reference Survey (HRS; Boselli et al. 2010a) is a quasi-stellar-mass-selected sample of 323 local galaxies. Various HRS studies have derived scaling relations between the gas, dust and star formation properties as well as trends with FIR/submm and UV colours, stellar mass, morphology and environment. Next to providing benchmark scaling relations, these works found that cluster galaxies are characterized by a significantly lower atomic, molecular and dust mass content than similar stellar mass galaxies in the field (Cortese et al. 2011, 2012a,b, 2014; Boselli et al. 2012, 2013, 2014b, 2015). The Herschel Astrophysical Terahertz Large Area Survey (H-ATLAS; Eales et al. 2010) is a blind, large-area submm survey which provides an unbiased and unrivalled view of

\footnotetext{
${ }^{1}$ Herschel is an ESA (European Space Agency) space observatory with science instruments provided by European-led Principal Investigator consortia and with important participation from NASA.
}

the nearby dusty Universe. Dust scaling relations in H-ATLAS have been studied by Bourne et al. (2012) through stacking 80 000 optically selected galaxies and also by Smith et al. (2011), who used fits to the UV-FIR photometry of $1402250 \mu \mathrm{m}$ selected sources. More recently, H-ATLAS has produced a local volume-limited sample, and Clark et al. (2015, hereafter C15) used it to study the dust properties of the first dust-mass-selected sample of galaxies in the local Universe.

C15 show that stellar-mass-selected samples are biased towards galaxies that have converted a lot of their gas into stars, i.e. towards more evolved galaxies, and thus under-represent immature high gas fraction sources. Dust selection produces a more uniform range of gas fractions but preferentially samples galaxies near the peak of their dust content. In this work, we compare a local, $\mathrm{H}_{\mathrm{I}}$ selected sample from the H-ATLAS equatorial fields to these stellarand dust-mass-selected samples. We will highlight scaling relations concerning dust properties as these have not been studied before

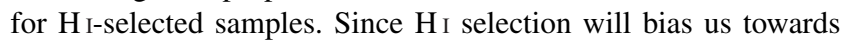
galaxies with high gas fractions, we can populate the scaling relations for these hitherto missing immature galaxies and, for the first time, study their dust properties. By comparing the three samples selected by stellar, dust and atomic gas content, we span a large range of gas fractions and can study the relationship of dust, gas and stars across as wide a range of evolutionary status as possible.

This paper is organized as follows. In Section 2, we describe the observations, the sample selection, the extended source photometry pipeline and the MAGPHYS SED fitting code that was used to obtain the galactic properties. In Section 3, we discuss the different surveys used in this work. In Section 4, we compare the dust, gas and stellar content of the $\mathrm{HI}$, dust- and stellar-mass-selected samples. In Section 5, we study the evolution of the star formation efficiency and in Section 6 we investigate the dust heating. Finally in Section 7, we study the obscuration for the different samples. We adopt the cosmology of Planck Collaboration I (2014), specifically $H_{0}=67.30 \mathrm{~km} \mathrm{~s}^{-1} \mathrm{Mpc}^{-1}, \Omega_{m}=0.315$ and $\Omega_{\Lambda}=0.685$.

\section{THE DATA}

In order to obtain a sample of galaxies with sufficient multiwavelength information to determine the stellar, dust and atomic gas (H I) content, it is necessary to select an area of sky which has been surveyed in the optical, in the submm and at $21 \mathrm{~cm}$. The ideal fields with the necessary multiwavelength data are the three equatorial fields $\left(\sim 160 \mathrm{deg}^{2}\right)$ of the H-ATLAS (Eales et al. 2010) which have excellent multiwavelength auxiliary data and overlap with the Galaxy And Mass Assembly spectroscopic survey (GAMA; Driver et al. 2009). The H I Parkes All-Sky Survey (HIPASS; Barnes et al. 2001), supplemented by the Arecibo Legacy Fast ALFA Survey (ALFALFA; Giovanelli et al. 2005), is used to determine the atomic gas properties.

\subsection{Observations}

HIPASS (Barnes et al. 2001; Meyer et al. 2004) provides $21 \mathrm{~cm}$ coverage over the equatorial H-ATLAS/GAMA fields. The Parkes beam size is 15.5 arcmin; the velocity resolution is $18 \mathrm{~km} \mathrm{~s}^{-1}$; and the rms noise is $13 \mathrm{mJy}$ beam $^{-1}$ in a channel of this width. The HIPASS catalogue (HICAT; Meyer et al. 2004; Zwaan et al. 2004; Wong et al. 2006) is used to identify our sources and extract the basic H I-parameters.

The HIPASS data are supplemented by observations from the ALFALFA (Giovanelli et al. 2005; Haynes et al. 2011; Haynes, 
private communication). With a beam size of $\sim 3.5$ arcmin and an rms noise of $\sim 2 \mathrm{mJy}^{-1}$ beam $^{-1}$ (for $11 \mathrm{~km} \mathrm{~s}^{-1}$ channels), ALFALFA outperforms HIPASS in both sensitivity and resolution. It does not, however, have full coverage over the three equatorial $\mathrm{H}$ ATLAS/GAMA fields in this study. For this reason, we use HIPASS data supplemented with ALFALFA where available.

The uniqueness and strength of this $\mathrm{H}$-selected sample is that it makes use of the H-ATLAS - the largest extragalactic submm survey covering $\sim 600 \mathrm{deg}^{2}$ in five bands from 100 to $500 \mu \mathrm{m}$. The H-ATLAS observations were carried out in parallel mode using the Photodetector Array Camera and Spectrometer (PACS; Poglitsch et al. 2010) and the Spectral and Photometric Imaging REceiver (SPIRE; Griffin et al. 2010) instruments on board the Herschel Space Observatory. This work makes use of the H-ATLAS Phase 1 public data release, hereafter 'DR1' (Bourne et al. 2016; Valiante et al. 2016). [More details on the H-ATLAS data reduction can be found in Valiante et al. (2016).] To determine counterparts to our $\mathrm{H}$ I-selected sources, we use the DR1 catalogue of $4 \sigma$ detections at $250 \mu \mathrm{m}$ (Valiante et al. 2016) produced using the MAD-X algorithm (Maddox et al., in preparation). Optical counterparts to H-ATLAS sources were found by direct comparison with the Sloan Digital Sky Survey Data Release 7 (SDSS DR7; Abazajian et al. 2009) and DR9 (Ahn et al. 2012) by means of matching H-ATLAS sources to SDSS objects within a 10 arcsec radius using a likelihood ratio technique, where only SDSS sources with a reliability of $R>0.8$ are considered to be likely matched to the H-ATLAS sources (Smith et al. 2011; Bourne et al. 2016).

For ultraviolet, optical and near-infrared (NIR) data, we use images compiled by the GAMA (Driver et al. 2011; Hopkins et al. 2013; Liske et al. 2015). GAMA provides spectroscopic redshifts, along with supplementary reductions of UV GALEX (Morrissey et al. 2007; Seibert et al. 2012), optical SDSS DR6 (AdelmanMcCarthy et al. 2008), NIR VISTA VIKING (Sutherland 2012) and mid-infrared (MIR) WISE (Wright et al. 2010; Cluver et al. 2014) data. Details of these reprocessed maps can be found in Driver et al. (2016).

Unfortunately, we do not have $\mathrm{CO}$ data for our $\mathrm{H}$ I-selected sample, so we cannot measure the molecular gas mass present in these galaxies. However, we have estimated molecular gas masses for our sources based on scaling relations from Saintonge et al. (2011) and Bothwell et al. (2014). We found that the estimated molecular gas component is small compared to the $\mathrm{H}$ I masses for $\mathrm{H}$ I-selected sources. Using these scaling relations to derive total $\left(\mathrm{H} \mathrm{I}+\mathrm{H}_{2}\right)$ gas masses instead of $\mathrm{H}$ I masses does not change the overall conclusions presented in this work.

\subsection{Sample selection}

Our sample consists of the 32 sources in the HICAT that overlap with the H-ATLAS/GAMA footprints. These H I sources are crossmatched to the H-ATLAS DR1 catalogue and to SDSS DR9 (Ahn et al. 2012). The matching is done by identifying all optical and submm sources that lie within the 15.5 arcmin Parkes beam and have spectroscopic redshifts within the redshift range of the HIPASS profile. To be accepted, optical matches need to have a reliable redshift from GAMA or SDSS and the H-ATLAS matches need to have a reliable SDSS counterpart $(R>0.8$; Smith et al. 2011). The H-ATLAS matches are combined with their corresponding optical matches when possible.

We identified two additional sources by checking the literature for bright $\mathrm{H}_{\text {I }}$ sources that are located in the H-ATLAS fields, but are not found by HIPASS. Both UGC0700 (Sulentic \& Arp 1983) and
NGC5746 (Popping \& Braun 2011) were detected with Arecibo (3.5 arcmin beam) and both are bright enough to be detected by HIPASS, yet lie just outside the beam of the closest HIPASS source (separation of about 20 arcmin). The close proximity to another bright HIPASS source likely caused these sources to be missed. However, they are still bright enough to meet our selection ${ }^{2}$ and both these sources are added to our sample.

Multiple optical matches are found for 30 out of the 32 HIPASS sources due to two different issues. The first is that the SExtractor (Bertin \& Arnouts 1996) source detection used by SDSS and GAMA was not optimized for the very extended local sources in our sample (semimajor axis up to 3 arcmin). Most of the sources in our sample also have clumpy optical distributions, which together with their large angular sizes, lead to SExtractor 'shredding' galaxies into several components; 71 per cent of the sources in our sample are affected by this shredding. For these sources, we determine the correct central position manually and reject the spurious listings. Fluxes were measured as described in Section 2.3.

After correcting the shredding issue, there are still a number of HIPASS sources that have multiple distinct galaxy matches. These galaxies are 'confused' in the large $\mathrm{H}$ I beam and there is no sure way of determining how much of the $\mathrm{H}$ i signal corresponds to each of the sources without obtaining higher angular resolution observations. The galaxies for which the HIPASS signal is confused are labelled ' $\mathrm{a}$ ' in Tables B1 and B2. The projected physical distance between these confused galaxies is relatively small $(\sim 100 \mathrm{kpc})$ and they form groups (consisting of up to five sources). In total, there are 49 matches to the 32 HIPASS sources in the sample.

In order to better determine the H I properties for the confused sources, we have supplemented the HIPASS data with ALFALFA data when available ( $3.5^{\prime}$ beam size). ALFALFA only covers the more northern sources in the sample (Dec. $>-0.05$ ) and we find an unconfused $\mathrm{H}$ I source for 23 out of the 49 optical matches. Because of its higher sensitivity, we use the ALFALFA H I measurements for all sources that lie in its footprint. The ALFALFA data resolve three of the nine confused HIPASS sources into seven separate $\mathrm{H}_{\mathrm{I}}$ sources, each with its own optical counterpart. We are then left with 6 confused HIPASS sources, containing 14 optical matches between them. For these, we have searched the literature for the highest resolution $21 \mathrm{~cm}$ observations available, leading to the deconfusion of five HIPASS sources into eight separate $\mathrm{H}_{\text {I }}$ sources with optical counterparts (see Table B2 for relevant references). This leaves us with one HIPASS source for which there are two optical matches. For this source, we estimate which of the two candidate counterparts contributes the vast majority of the H I signal. The dominant source was chosen by comparing the stellar masses, the near-ultraviolet (NUV) $-r$ colours and the offsets in optical positions and velocities of the optical counterparts from HIPASS. Based on these, we are confident that one counterpart (labelled ' $b$ ' in Tables B1 and B2) has nearly all the H I mass, and the other is a small satellite galaxy that can be discarded together with all the other optical matches without Hi detections. In Table 1, we present the key characteristics of the sources in our Hi-selected sample, such as their common names, positions, redshifts, distances and sizes. Distances were calculated using spectroscopic redshifts using $H_{0}=67.30 \mathrm{~km} \mathrm{~s}^{-1} \mathrm{Mpc}^{-1}$, with velocities corrected by GAMA (Baldry et al. 2012) to account for

\footnotetext{
${ }^{2}$ Without the close proximity to another HIPASS source, both these sources would have been included in the HIPASS sample.
} 
Table 1. Properties of the $40 \mathrm{HIGH}$ sources derived with MAGPHYS SED fitting. The columns are (from left to right): index, galaxy name, stellar mass, dust luminosity, dust mass, $M_{\mathrm{d}} / M_{*}$, temperature of the cold dust component, SFR averaged over the last $10^{8} \mathrm{yr}$, SSFR averaged over the last $10^{8} \mathrm{yr}, f_{\mu}$ (the fraction of the total dust luminosity contributed by the diffuse ISM) and the FUV attenuation by dust. Uncertainties are indicated by the 84th to 16th percentile range from each of the individual PDF.

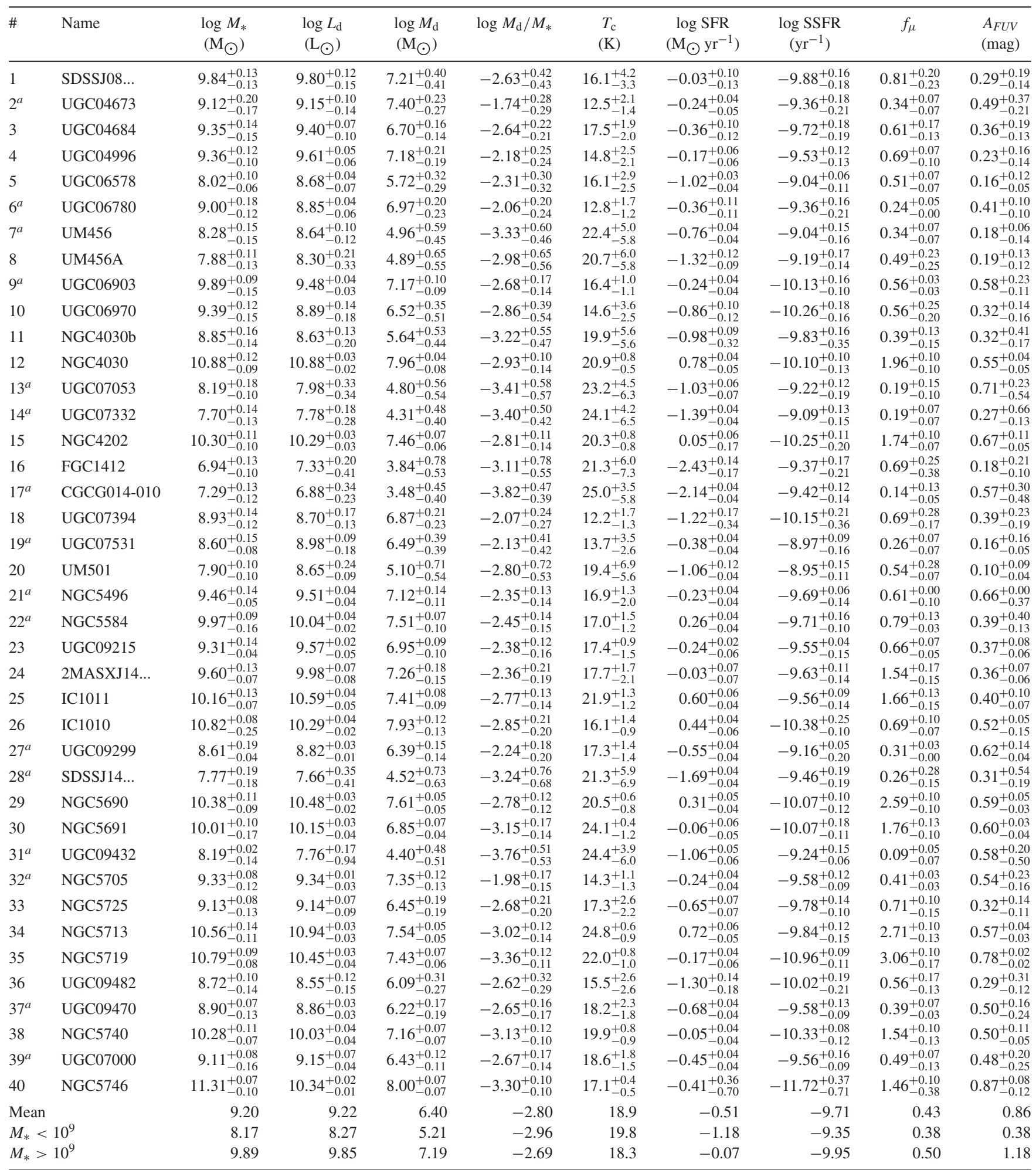

Notes. ${ }^{a}$ For these sources, we use SFR and SSFR estimates using the same method for SFR as C15 since the MAGPHYS SFR and SSFR PDF show two peaks. The two peaks occur because the model SFR (averaged over the last $10^{8} \mathrm{yr}$ ) will be quite different if it includes a burst (that ended nearly $10^{8} \mathrm{yr}$ ago), compared to if the burst ended just before $10^{8} \mathrm{yr}$ ago, even though there are only very small differences to the SEDs. Schofield et al. (in preparation) will explore this issue in more detail. We note that the C15 SFR estimates would be biased when the SSFR is small and the dust luminosity has a large contribution from heating by old stars (Boquien et al. 2016). This is not the case for these galaxies. 
Table 2. The Spearman rank correlation coefficients $(r)$ and lines of best fit $(y=a x+b$, where $a$ is the slope and $b$ is the intercept) for the significant correlations in the form of a power law. The best-fitting relations were determined using a BCES linear regression method (Akritas \& Bershady 1996) using the HIGH, HAPLESS and HRS samples combined. For HRS, only LTGs are included (both H i deficient and H I normal). The first two columns specify the $x$ and $y$ parameters, the last columns specify whether HIGH-low is offset and whether the derived relation is dependent on the selection used. We caution the use of relations which are strongly dependent on the selection criteria. HiGH-low is not included in the combined sample if it is offset (lower dust mass) compared to the other samples.

\begin{tabular}{|c|c|c|c|c|c|c|}
\hline$y$ & $x$ & $r$ & Slope & Intercept & $\begin{array}{l}\text { HIGH-low } \\
\text { offset }\end{array}$ & $\begin{array}{l}\text { Strong selection } \\
\text { dependence }\end{array}$ \\
\hline $\log M_{\mathrm{H}_{\mathrm{I}}} / M_{*}$ & $\log M_{*}$ & -0.59 & $-0.69 \pm 0.05$ & $6.02 \pm 0.47$ & & $\sqrt{ }$ \\
\hline $\log M_{\mathrm{H}_{\mathrm{I}}} / M_{*}$ & NUV-r & -0.84 & $-0.65 \pm 0.03$ & $1.30 \pm 0.09$ & & \\
\hline $\log M_{\mathrm{d}} / M_{*}$ & $\log M_{*}$ & -0.44 & $-0.26 \pm 0.03$ & $-0.44 \pm 0.34$ & $\sqrt{ }$ & $\sqrt{ }$ \\
\hline $\log M_{\mathrm{d}} / M_{*}$ & $\log$ SSFR & 0.72 & $0.51 \pm 0.03$ & $2.30 \pm 0.30$ & $\sqrt{ }$ & \\
\hline $\log M_{\mathrm{d}} / M_{*}$ & NUV-r & -0.77 & $-0.33 \pm 0.02$ & $-1.92 \pm 0.06$ & $\sqrt{ }$ & \\
\hline $\log M_{\mathrm{d}} / M_{*}$ & $\log M_{\mathrm{H}_{\mathrm{I}}} / M_{*}$ & 0.87 & $0.47 \pm 0.02$ & $-2.59 \pm 0.02$ & $\sqrt{ }$ & \\
\hline $\log M_{\mathrm{d}} / M_{\mathrm{H}}$ & $\log M_{*}$ & 0.47 & $0.32 \pm 0.04$ & $-5.33 \pm 0.37$ & $\sqrt{ }$ & $\sqrt{ }$ \\
\hline $\log M_{\mathrm{d}} / M_{\mathrm{H}}$ & NUV-r & 0.66 & $0.28 \pm 0.02$ & $-3.06 \pm 0.07$ & $\sqrt{ }$ & \\
\hline $\log M_{\mathrm{d}} / M_{\mathrm{HI}}$ & $\log M_{\mathrm{H}_{\mathrm{I}}} / M_{*}$ & -0.86 & $-0.52 \pm 0.02$ & $-2.57 \pm 0.02$ & $\sqrt{ }$ & \\
\hline $\log \mathrm{SFR} / M_{d}$ & $\log M_{\mathrm{H}_{\mathrm{I}}} / M_{*}$ & 0.37 & $0.25 \pm 0.03$ & $-7.19 \pm 0.03$ & $\sqrt{ }$ & \\
\hline $\log \mathrm{SFR} / M_{\mathrm{H}_{\mathrm{I}}}$ & $\log M_{\mathrm{H}_{\mathrm{I}}} / M_{*}$ & -0.53 & $-0.29 \pm 0.03$ & $-9.80 \pm 0.02$ & & \\
\hline $\log \mathrm{SFR} / M_{\mathrm{H}_{\mathrm{I}}}$ & $\log \Sigma_{*}$ & 0.58 & $0.50 \pm 0.06$ & $-10.28 \pm 0.07$ & & \\
\hline
\end{tabular}

bulk deviations from Hubble flow ${ }^{3}$ (Tonry et al. 2000). We use the standard prescription to determine $\mathrm{H}$ I masses:

$M_{\mathrm{HI}}=2.356 \times 10^{5} S_{\text {int }} D^{2}$,

where $M_{\mathrm{HI}}$ is the $\mathrm{H}_{\mathrm{I}}$ mass in solar units, $S_{\text {int }}$ is the integrated $21 \mathrm{~cm}$ line flux in $\mathrm{Jy} \mathrm{km} \mathrm{s}^{-1}$ and $D$ is the Hubble-flow-corrected distance in Mpc. The $\mathrm{H}$ I-derived properties are listed in Table 2.

The $\mathrm{H}_{\mathrm{I}}$ fluxes and masses in Table B2 have not been corrected for self-absorption (which occurs if there is a high optical depth in the line of sight for $\mathrm{H}_{\mathrm{I}}$ clouds). Bourne et al. (2013) calculated this correction for a sample of 20 galaxies over the H-ATLAS fields. The average correction factor for the overlapping sources with our $\mathrm{HI}_{\mathrm{I}}$ selected sample is 1.09. Neither of the comparative samples used in this work (see Section 3) have been corrected for self-absorption. For this reason, and because of the uncertainty associated with the correction, we do not account for self-absorption in this work, but note that our gas masses, particularly for edge-on galaxies, could therefore be underestimated.

Adding in higher resolution $\mathrm{H}$ I data for known HIPASS detections could affect our $\mathrm{H}$ i selection. Although we have found ALFALFA counterparts to each of the HIPASS sources in the common region, we cannot be confident that these individual counterparts would have made the HIPASS detection limit by itself. This is possibly an issue for the three sources in our sample with $S_{\text {int }}<1.7 \mathrm{Jy}$ (labelled ' $c$ ' in Table 1) and we therefore ignore these when we discuss selection effects later. These sources do not change any of our conclusions, which is why we include them in our plots and do not discuss further. Finally, we arrive at a sample of 40 unconfused $\mathrm{H}$ I-selected sources, 22 of which overlap with the $\mathrm{C} 15$ dust-selected sample (Table B1). Note that this is more than the original number of HIPASS sources due to the additional ALFALFA and literature data for the confused sources. These 40 sources will form our sample of 'H I-selected Galaxies in H-ATLAS', hereafter referred to as the HIGH sample.

\footnotetext{
${ }^{3} \mathrm{C} 15$ used a redshift-independent distance for NGC5584 from measurements of Cepheid variables (Riess et al. 2011). However, there is a lot of scatter in redshift-independent distance estimates for NGC5584, and we have opted to use the same method as for the other sources in our sample.
}

\subsection{Extended-source photometry}

To study the extended galaxies in a consistent way across 21 bands ranging from far-ultraviolet (FUV) to $500 \mu \mathrm{m}$, we consider the same physical area for each wavelength. We perform our own aperturematched photometry across the entire UV-to-submm wavelength range, with exceptions for the IRAS $60 \mu \mathrm{m}$ measurements and for the PACS 100 and $160 \mu \mathrm{m}$ aperture fitting. The exceptions are described in Appendix A.

The first stage of the process consists of determining the appropriate aperture for each source. As described in $\mathrm{C} 15$, the optimal shape and size of the aperture are automatically determined in each band from FUV to $22 \mu \mathrm{m}$, and the largest aperture (after correcting the aperture size for the point spread function) is selected as the definitive photometric aperture (typically, FUV or NUV). The semimajor axes of the apertures used are listed in Table B1.

Next we removed bright foreground stars as described in C15, by using a curve of growth to measure the size of each star and then replacing stellar pixels with pixels randomly drawn from an annulus around the mask. In addition to this, a similar technique was used to remove background galaxies and remaining bright stars, yet using manually determined masking apertures. This was necessary for our $\mathrm{HI}$-selected sample as many of the most $\mathrm{H}$ I-rich sources have low surface brightness and are more susceptible to the effects of contaminating sources in the apertures.

After contaminant removal, the aperture-matched photometry was performed in each band and the uncertainties were determined. The photometry from the FUV to $K_{s}$ band was corrected for Galactic extinction in the same way as GAMA, using the method described in Adelman-McCarthy et al. (2008).

\subsubsection{Uncertainties}

The aperture noise for the UV-IR bands was estimated, as in C15, using random apertures placed on non-target regions of the map and using a clipping procedure to mimic the effects of the star subtraction performed in the main target aperture and sky annulus. The aperture noise for Herschel was also estimated by placing random apertures, yet without additional clipping. Instead all extended sources in the H-ATLAS DR1 catalogue were masked using the 
H-ATLAS aperture and only randomly placed apertures not containing masked pixels were accepted.

Compared to $\mathrm{C} 15$, we include an additional source of uncertainty related to the accuracy of the star subtraction process. For each source and each band, we calculate the uncertainty as the average relative difference between the original photometry and photometry performed with a contaminant removal with stellar/galaxy radii that differ by \pm 10 per cent. This uncertainty is small for most sources but can dominate the total uncertainty for the few $(\sim 10$ per cent of the sample) sources with strong stellar contamination.

The above errors are added in quadrature to the aperture noise and the resulting photometry is given in Table B3 in Appendix B. Before fitting SEDs, we apply an additional term of uncertainty to account for the calibration uncertainty, model uncertainties in our SED fitting (see the following section) and contributions from spectral lines. For this additional term, we use either 10 per cent or the calibration error, whichever is larger.

\subsection{SED fitting}

To interpret the resulting panchromatic SEDs of the galaxies in our HiGH sample in terms of their physical properties, we use the MAGPHYs code of da Cunha, Charlot \& Elbaz (2008). ${ }^{4}$ MAGPHYS uses libraries of $\sim 50000$ optical and $\sim 50000$ IR models to describe the stellar and dust SEDs, respectively. These models are combined in such a way that the energy balance is maintained in both the diffuse ISM and the birth clouds. For each combination, the model SED is compared to the observed galaxy SED and a goodness of fit $\chi^{2}$ calculated. Probability density functions (PDFs) can then be made for any of the model physical parameters by weighting the value of that parameter by the probability $\mathrm{e}^{-\chi^{2} / 2}$. Our most reliable estimate for each parameter is the median value of its PDF and the corresponding uncertainties are the 16th and 84th percentiles of the PDF. For more details on the MAGPHYS models, we refer to da Cunha et al. (2008).

We made some adaptations to MAGPHYS in order to tailor to our sample. These include the following:

(i) The cold dust temperature range needed to be extended to 10-30 K (instead of the standard 15-25 K) in order to fit some of the dusty sources in our sample.

(ii) Some $\mathrm{HIGH}$ sources have bands with low signal-to-noise ratio (SNR), and in some cases the measured fluxes in the FIR are negative, though with errors which are consistent with a zero or positive flux at the $1 \sigma$ level. The standard MAGPHYS version does not deal with negative fluxes, yet we have incorporated them in our $\chi^{2}$ calculation, as they still give statistical constraints.

(iii) Additionally, we added a routine that allows us to include IRAS $60 \mu \mathrm{m}$ upper limits (necessary for a third of our sample). For these upper limits, we only add a contribution to $\chi^{2}$ if the model fluxes are higher than the upper limit flux.

(iv) We have generated a PDF for the dust attenuation in the FUV $\left(A_{\mathrm{FUV}}\right)$ by comparing the attenuated and unattenuated model FUV fluxes for each model.

Before fitting the SEDs, we correct the SPIRE fluxes in Table B3 for $K_{\text {beam }}$ and apply the additional uncertainty term to all bands (see Section 2.3.1). MAGPHYS intrinsically applies colour corrections to all fluxes, so the $K_{\text {colP }}$ colour corrections from the SPIRE handbook

\footnotetext{
${ }^{4}$ The MAGPHYS package containing the models of da Cunha et al. (2008) is publicly available at: http://www.iap.fr/MAGPHYs.
}

do not need to be applied. The MAGPHYs results for the HIGH sample are presented in Table 1, and multiwavelength images and SEDs for $\mathrm{HIGH}$ are given in Figs B1 and B2, respectively.

\section{SURVEYS USED IN THIS WORK}

Here, we introduce the dust-selected and stellar-mass-selected samples of local galaxies to compare with our $\mathrm{H}$ I-selected sample.

\subsection{Dust-selected sample}

The best comparative dust-selected sample is the Herschel-ATLAS Phase-1 Limited-Extent Spatial Survey (HAPLESS) described in the companion paper to this work (C15). HAPLESS is a volumelimited sample consisting of $42 \mathrm{H}$-ATLAS galaxies detected at $250 \mu \mathrm{m}$ in the equatorial H-ATLAS fields with $0.0035<z<0.01$. Throughout the rest of this work, we will refer to HAPLESS as a dust-selected sample to indicate this $250 \mu \mathrm{m}$ flux selection.

HAPLESS has 22 sources in common with $\mathrm{HIGH}$, and the photometry was performed using the same pipeline. For consistency, we have redetermined the Herschel photometry for HAPLESS using the H-ATLAS DR1 maps that have since become available. Additionally, we redetermined the galaxy properties for HAPLESS using MAGPHYS instead of the combination of different techniques at different wavelengths used by C15. The MAGPHYs cold dust temperatures are, on average, $3 \mathrm{~K}$ warmer and the dust masses smaller by 0.25 dex than the results in $\mathrm{C} 15$, and the offset is largest for sources with cold $\left(T_{\mathrm{c}}<15 \mathrm{~K}\right)$ dust temperature in $\mathrm{C} 15$. The differences originate in part from differences in the SED fitting technique and in part from changes to the Herschel photometry due to using the H-ATLAS DR1 data release instead of Phase 1 version 3. In contrast to modified blackbody fits in C15, MAGPHYs limits the warm dust to $30 \mathrm{~K}<T_{\mathrm{w}}<60 \mathrm{~K}$, and at least half of the dust luminosity in the diffuse ISM must originate from the cold dust component. Therefore, MAGPHYS assigns low probabilities to poorly constrained cold dust components that make up a tiny fraction of the total luminosity but peak at the longest wavelengths, therefore making up the bulk of the dust mass. Additionally, MAGPHYs uses the median $T_{\mathrm{c}}$ from the PDF whereas $\mathrm{C} 15$ used the best fit to the data; when comparing $\mathrm{C} 15$ with the best-fitting MAGPHYS result, we find overall a better agreement between the two estimates. C15 compiled literatures of atomic gas masses, including HIPASS (Meyer et al. 2004) and ALFALFA (Haynes, private communication).

\subsection{Stellar-mass-selected sample}

For a stellar-mass-selected sample, we follow C15 and use the HRS (Boselli et al. 2010a) which targeted 323 local galaxies. The HRS is a volume-limited sample (between 15 and $25 \mathrm{Mpc}$ ) and uses $K_{s}$-band selection because this band suffers least from extinction and is known to be a good proxy for stellar mass; throughout the rest of this work, we will refer to the HRS as a stellar-mass-selected sample. The HRS contains both late-type galaxies (LTGs) and 75 early-type galaxies (ETGs), and includes many galaxies in cluster environments. We do not include ETGs when determining bestfitting relations and correlations. Instead, we highlight them as a separate subsample in the plots.

Again, for consistency, we derived properties for HRS sources using MAGPHYS. Our results are slightly different from the MAGPHYS HRS results in Viaene et al. (2016) because they did not apply any corrections for Galactic extinction and $K_{\text {beam }}$, and used smaller uncertainties. For the HI masses of the HRS galaxies, we used 


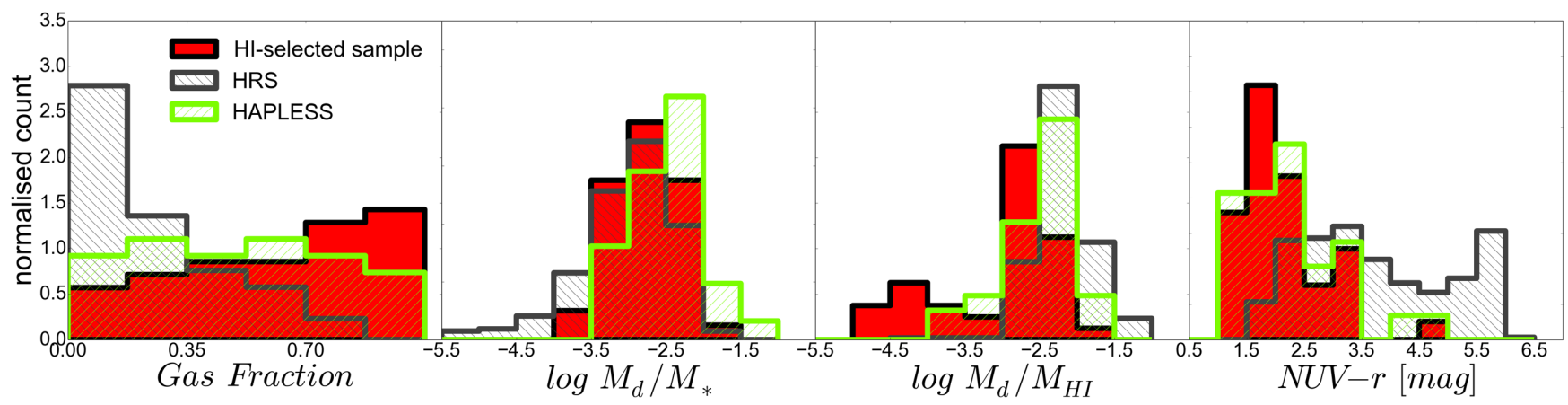

Figure 1. From left to right: histogram showing the distribution of gas fraction $\left(\frac{M_{\mathrm{H}_{1}}}{M_{*}+M_{\mathrm{H}_{\mathrm{I}}}}\right)$, specific dust mass, dust-to-H I ratio and NUV $-r$ colour (proxy for SSFR) for the HIGH sample, HAPLESS and HRS.
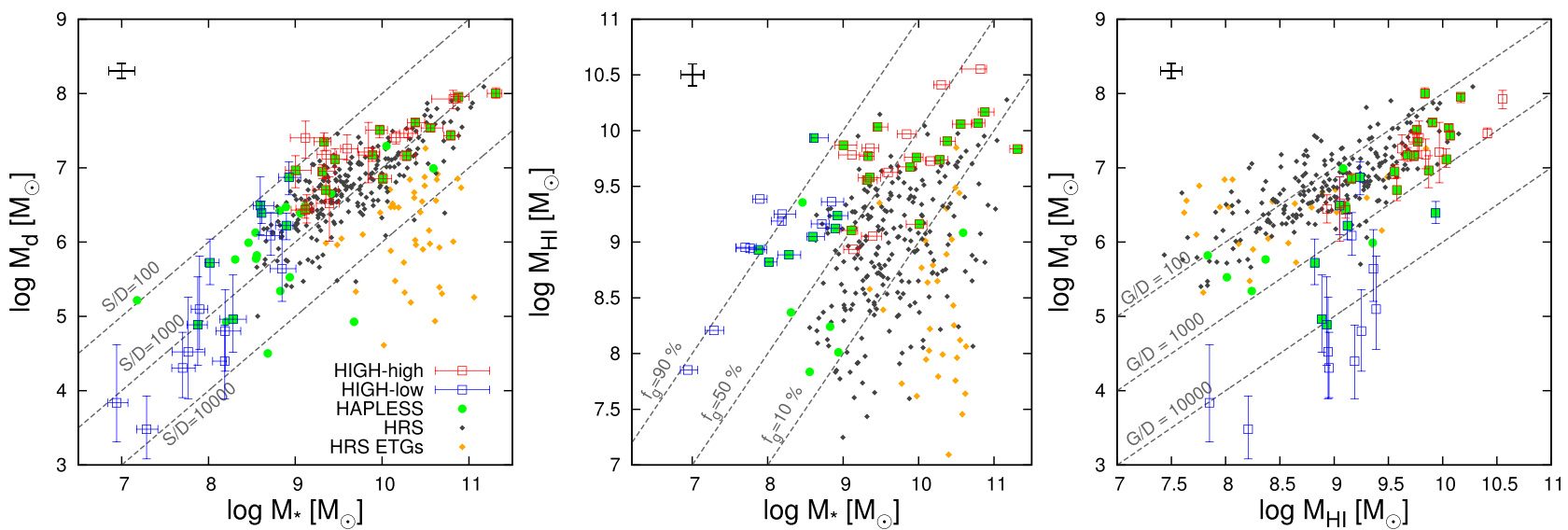

Figure 2. Left: scaling relations between dust and stellar mass. Centre: H I versus stellar mass. Right: dust versus $\mathrm{H}_{\mathrm{I}}$ mass. For each plot, a representative error bar for HRS is shown in the upper-left corner. Note the selection effects towards higher dust and gas masses for the HI-selected HIGH-low (blue squares), HIGH-high (red squares) samples and the dust-selected HAPLESS sample (green circles) compared to the stellar-mass-selected HRS sample (grey dots). The common sources between HAPLESS and our $\mathrm{H}$ I-selected subsamples are shown as green filled squares with red/blue borders. Lines of constant $M_{*} / M_{\mathrm{d}}(\mathrm{S} / \mathrm{D})$, gas fraction $\left(f_{\mathrm{g}}\right)$ and $M_{\mathrm{H}_{\mathrm{I}}} / M_{\mathrm{d}}(\mathrm{G} / \mathrm{D})$ are shown in grey.

the unconfused results from Boselli, Cortese \& Boquien (2014a). For HRS, CO-derived $\mathrm{H}_{2}$ masses are presented in Boselli et al. (2014a) and can be relatively large compared to their H I. However, they are still small compared to the total baryon mass, and using total $\left(\mathrm{H}+\mathrm{H}_{2}\right)$ gas masses instead of $\mathrm{H}_{\mathrm{I}}$ masses only gives small differences to the overall scaling relations for HRS in this work.

\section{DUST, GAS AND STARS}

We first investigate the distribution of the relative masses of stars, dust and atomic gas. The distribution of the gas fractions $\left(\frac{M_{\mathrm{H}_{\mathrm{I}}}}{M_{+}+M_{\mathrm{H}}}\right)$ in the left-hand panel of Fig. 1 shows that the HiGH sample is more gas-rich than the HRS, while the HAPLESS gas fractions are relatively uniformly distributed. In this paper, we will define the evolutionary status of a galaxy using its gas fraction as a measure of how much of the available gas reservoir has been converted into stars. ${ }^{5}$ HRS then consists mainly of evolved sources; HAPLESS consists of galaxies at a range of stages of evolution; and the $\mathrm{HIGH}$ sample consists mainly of relatively unevolved sources. Our $\mathrm{HI}$ selection therefore gives us vital insights into the 'youthful' sources which were previously under-represented in samples like the HRS.

\footnotetext{
${ }^{5}$ The most important caveat to this method is that we do not take into account interactions like inflows, outflows and merging.
}

Fig. 1 also shows that the specific dust mass $\left(M_{\mathrm{d}} / M_{*}\right)$ is highest for HAPLESS, followed by HIGH and then HRS. When we look at the distribution of dust-to-H I ratio, we now find that $\mathrm{HIGH}$ has the lowest $M_{\mathrm{d}} / M_{\mathrm{H}}$, followed by HAPLESS and then HRS. Finally, we show the NUV $-r$ colour distribution in the right-hand panel of Fig. 1. This colour is closely related to the SSFR and we find that both the HAPLESS and $\mathrm{HIGH}$ samples are much bluer and thus more actively forming stars than the HRS. The large tail of red sources in HRS is because it contains a larger fraction of more evolved, passive sources.

The HiGH sample consists mainly of very blue, low surface brightness gas-rich sources which have irregular or flocculent morphologies and are actively forming stars. The blue sources in our sample are divided into dust-rich and dust-poor subsamples at $M_{*} \sim 10^{9} \mathrm{M}_{\odot}$ (see also Fig. 2). The blue dust-rich sources were already discussed in $\mathrm{C} 15$ and constitute around half of all dustmass-selected galaxies in the local volume. In the rest of this work, we will highlight the new population of blue dust-poor sources as a separate subsample, using a stellar mass cut of $M_{*}<10^{9} \mathrm{M}_{\odot}$. We will use this criterion to split the $\mathrm{HIGH}$ sample into $\mathrm{HIGH}$-low (for $M_{*}<10^{9} \mathrm{M}_{\odot}$ ) and $\mathrm{HIGH}-$ high (for $M_{*}>10^{9} \mathrm{M}_{\odot}$ ) throughout the rest of this work.

In Fig. 2, we show the stellar, dust and $\mathrm{H}$ I mass scaling relations. In the left-hand panel, we find a strong correlation between dust and 
stellar masses for both $\mathrm{HIGH}$ (Spearman rank correlation coefficient $r=0.93)$ and HAPLESS $(r=0.81)$. For HRS, there is a strong correlation $(r=0.88)$ for the LTGs, yet the correlation weakens significantly $(r=0.30)$ if the ETGs are included. Comparing the $\mathrm{H}$ I and stellar masses (the centre panel of Fig. 2), we find the strongest correlation for $\mathrm{HIGH}(r=0.77)$, a weaker one for HAPLESS $(r=$ $0.67)$ and the weakest for the HRS LTGs $(r=0.63)$. When the ETGs are included, there is no significant correlation for HRS. The HRS and the $\mathrm{HIGH}$ sample segregate in this plot because they intrinsically consist of galaxies in different stages of evolution (stellar mass selection favours lower gas fractions and vice versa). In the righthand panel of Fig. 2, we find a strong correlation between the $\mathrm{H}_{\mathrm{I}}$ and the dust mass for the HAPLESS, the HRS and the HIGH-high sample (Spearman rank coefficient of $r=0.74$ for the combined samples). However HiGH-low lies significantly below this relation and we will investigate the reasons for this in the following sections. Interestingly, The HRS ETGs now follow the same trend as the LTGs. For a given H I mass, HAPLESS and HIGH have lower dust masses on average than the HRS.

\subsection{Gas richness and specific star formation rate}

Fig. 3 shows how gas richness $\left(M_{\mathrm{H}_{\mathrm{I}}} / M_{*}\right)$ scales with stellar mass and NUV $-r$, which is known to be a good proxy for SSFR (e.g. Schiminovich et al. 2007). These relations have been extensively studied for HRS (Cortese et al. 2011), ALFALFA (Huang et al. 2012), H $\alpha 3$ (Gavazzi et al. 2013), GASS (Catinella et al. 2013) and in earlier work (Gavazzi \& Scodeggio 1996; Boselli et al. 2001). As seen in Fig. 3, the HiGH sample follows the same relations as determined for other $\mathrm{H}$ I-selected samples, such as ALFALFA.

Some of the selection effects for the different samples are evident in the top panel. The Hi selection of HiGH (and ALFALFA) selects higher $M_{\mathrm{H}_{\mathrm{I}}} / M_{*}$ at fixed $M_{*}$ compared to the stellar mass selection of HRS. This is due both to the Hi selection favouring gas-rich galaxies (and vice versa for stellar mass selection), and also in part to a fraction ( $\sim 25$ per cent) of the HRS sources being in the Virgo cluster. In Fig. 3, we have used open symbols for H I-deficient LTGs in HRS. Following Cortese et al. (2011), we consider galaxies to be $\mathrm{H}_{\mathrm{I}}$ deficient if $\operatorname{Def}_{\mathrm{H}_{\mathrm{I}}} \geq 0.5$ (this corresponds to galaxies with 70 per cent less hydrogen than isolated systems with the same diameter and morphological type). Next to our best-fitting relation for all samples combined (excluding ETGs), we have also plotted the best-fitting line excluding $\mathrm{H}_{\mathrm{I}}$-deficient galaxies to illustrate the effect of including $\mathrm{H}$ I-deficient galaxies in scaling relations.

We have also added the HIPASS detection limits at $M_{*}=$ $10^{7.5} \mathrm{M}_{\odot}$ and $M_{*}=10^{8} \mathrm{M}_{\odot}$ as black arrows to Fig. 3 (top), assuming a distance equal to the average distance for HiGH-low (29.2 Mpc). The lack of sources below the dashed line at low $M_{*}$ is due to this limit. However, the upper bound of the trend in Fig. 3 does not suffer from these selection effects. The large range of gas fractions found at a given stellar mass indicates that, although the star formation history has a well-known dependence on halo mass (more massive galaxies are more evolved; e.g. Cowie et al. 1996), local factors such as environment and gas supply play an important role (e.g. De Lucia et al. 2006) and thus cause the scatter in the top panel of Fig. 3. Here, we clearly see that the $M_{\mathrm{H}_{\mathrm{I}}} / M_{*}$ versus $M_{*}$ scaling relation fit depends on the sample used. The relation for HRS (or any stellar-mass-selected sample) is offset to that derived for an $\mathrm{HI}$-selected sample. It is also sensitive to the environment, with samples from high-density regions lying below the scaling relations.
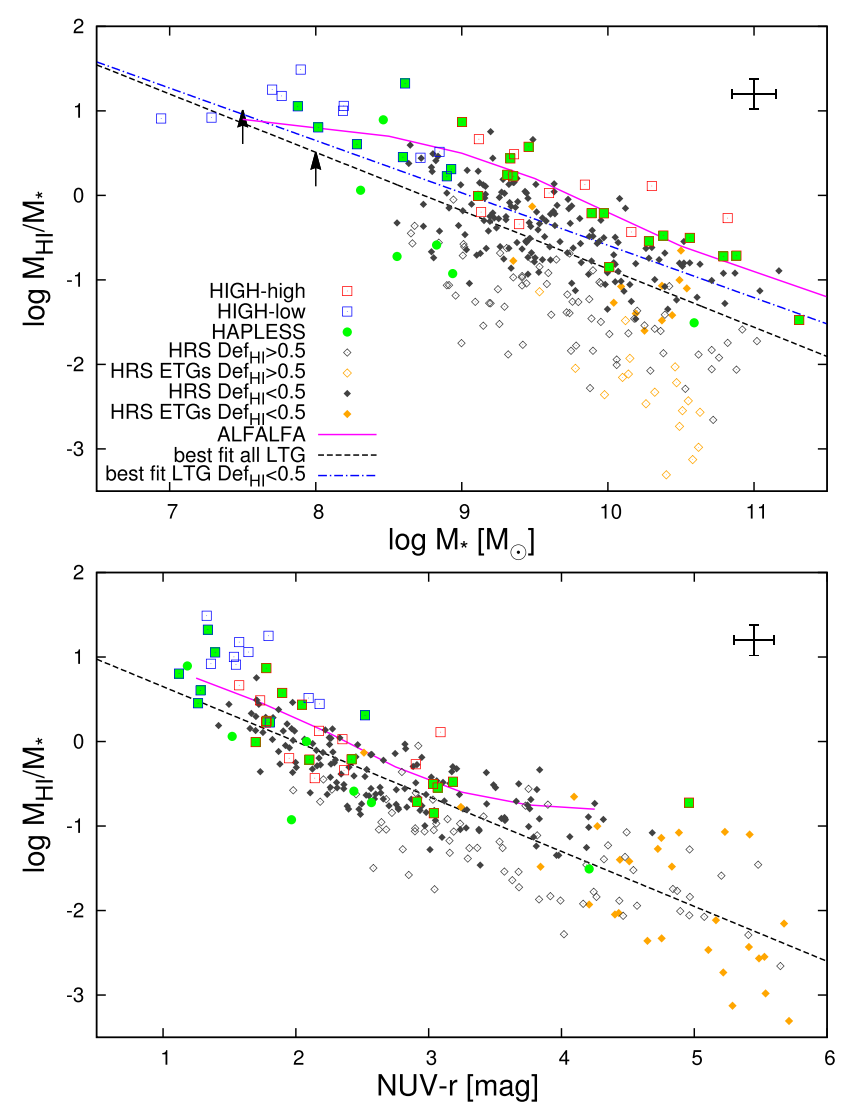

Figure 3. Trends with $M_{\mathrm{H}_{\mathrm{I}}} / M_{*}$ and SSFR. Symbols are as in Fig. 2, with open symbols for $\mathrm{H}_{\mathrm{I}}$-deficient $\left(\mathrm{Def}_{\mathrm{H}_{\mathrm{I}}} \geq 0.5\right.$ ) LTGs in HRS and filled symbols for H I normal HRS LTGs. The best-fitting power-law relationship for the combined samples (excluding ETGs) is shown as a black dashed line, and the best relation also excluding $\mathrm{H}$ I-deficient HRS galaxies as a blue dash-dot line. Top: $M_{\mathrm{H}_{\mathrm{I}}} / M_{*}$ against stellar mass. The ALFALFA relation (Huang et al. 2012) is shown in magenta. The HIPASS detection limits at $M_{*}=10^{7.5} \mathrm{M}_{\odot}$ and $M_{*}=10^{8} \mathrm{M}_{\odot}$ are shown as black arrows. Bottom: $M_{\mathrm{H}} / M_{*}$ against NUV $-r$ colour (proxy for SSFR). This strong correlation is applicable to all samples and thus is a very useful scaling relation.

Fig. 3 (bottom) shows that the gas fraction is more strongly correlated with NUV $-r$ colour than with $M_{*}(r=-0.84$ and $r=-0.59$, respectively, for all samples combined). The different samples collated here (including the $\mathrm{H}_{\mathrm{I}}$-deficient sources) now lie on the same best-fitting relation (contrary to the top panel). The range in gas fraction at fixed NUV $-r$ is thus significantly smaller than at fixed $M_{*}$. This can be understood by realizing that the parameters on both axes scale with the cold gas content, and this shows that $M_{\mathrm{H}} / M_{*}$ is a strong driver of the SSFR (either directly or indirectly through scaling relations with the molecular gas, which is directly involved in star formation; e.g. Bigiel et al. 2011; Saintonge et al. 2011; Schruba et al. 2011; Bothwell et al. 2014). This is in line with the scatter in the main sequence of star-forming galaxies (SSFR versus $M_{*}$ ) being driven by the gas supply (Catinella et al. 2010; Cortese et al. 2011; Huang et al. 2012; Santini et al. 2014). We have checked that this is the case for our samples by colour-coding data by $\mathrm{H}_{\mathrm{I}}$ mass in the relation between SSFR and $M_{*}$.

\subsection{Specific dust scaling relations}

Cortese et al. (2012a) and Bourne et al. (2012) have studied specific dust $\left(M_{\mathrm{d}} / M_{*}\right)$ scaling relations for HRS and for H-ATLAS stacks 

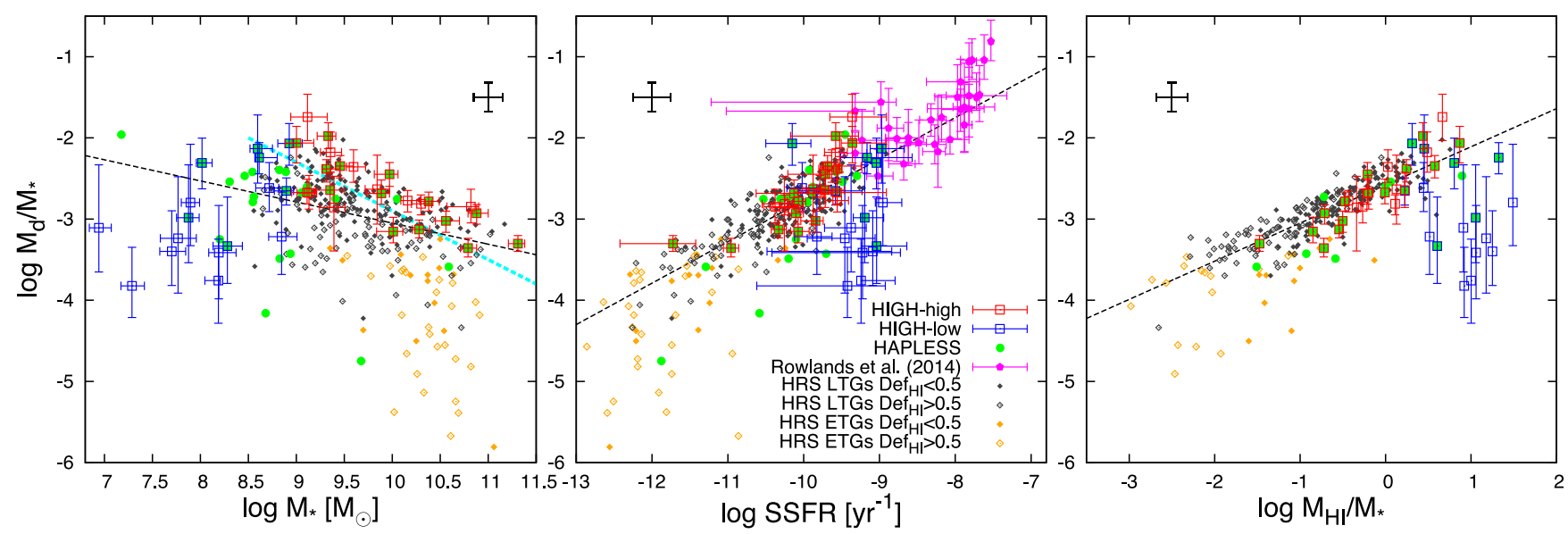

Figure 4. $M_{\mathrm{d}} / M_{*}$ scaling relations for the two $\mathrm{H}$ I-selected subsamples, HRS and HAPLESS. From left to right: Scaling relations with stellar mass, SSFR and $M_{\mathrm{H}} / M_{*}$. Correlations are found for each of the scaling relations for the high stellar mass $\mathrm{H}$ I-selected sample, HAPLESS and the HRS and the line of best fit for these 'evolved' samples combined is shown as a black dashed line. The relation from Bourne et al. (2012) for blue optically selected galaxies is given as a cyan dashed line. The ETGs are not included in our best-fitting relations. The symbols are as in Fig. 3.

on optically selected sources, respectively. They found a strong anticorrelation between $M_{\mathrm{d}} / M_{*}$ and NUV $-r$ colour, and a weaker anticorrelation with stellar mass, similar to the scaling relations for $M_{\mathrm{H}_{\mathrm{I}}} / M_{*}$ in the previous section (see also da Cunha et al. 2010; Smith et al. 2012; Rowlands et al. 2014a). Viaene et al. (2014) also note a similar trend for regions inside M31, indicating that the driving processes for these scaling relations (most likely star formation) are local processes. Cortese et al. (2012a) also found a strong correlation of $M_{\mathrm{d}} / M_{*}$ with gas fraction. Fig. 4 shows the specific dust scaling relations for the different samples. We find that the scaling relations for HRS, HAPLESS and HiGH-high are consistent with those in Cortese et al. (2012a) and Bourne et al. (2012).

For HIGH-low however, we find that the sources lie significantly below the trends for the other samples in each of the scaling relations in Fig. 4. The benchmark dust scaling relations identified by Cortese et al. (2012a) and Bourne et al. (2012) $)^{6}$ based on optically selected samples, do not hold for gas-rich, low $M_{*}$ (unevolved) sources. We note that a larger sample is necessary to determine the appropriate relationship for these low $M_{*}$ sources.

For the HRS, HAPLESS and HIGH-high samples, we find that $M_{\mathrm{d}} / M_{*}$ correlates most strongly with $M_{\mathrm{H}_{\mathrm{I}}} / M_{*}(r=0.87)$, followed by SSFR $(r=0.72)$ and then stellar mass $(r=-0.44)$. For the scaling relations with stellar mass in Fig. 4 (left), we find an offset towards higher $M_{\mathrm{d}} / M_{*}$ for the HIGH-high and HAPLESS samples compared to HRS (similar to Fig. 3). This offset is absent in the scaling relations with SSFR and $M_{\mathrm{H}_{\mathrm{I}}} / M_{*}$, which appear to be the more fundamental parameters driving the specific dust mass.

In the centre panel of Fig. 4, we have plotted $M_{\mathrm{d}} / M_{*}$ against SSFR, and have added the sample of high-redshift submillimetre galaxies (SMGs) from Rowlands et al. (2014a), which were also fitted with MAGPHYS. These galaxies are forming stars at a remarkably high rate and lie on a relation that extends the trend for $\mathrm{HIGH}$-high, HAPLESS and the HRS (the best-fitting relation was not fitted to the SMGs). The correlation of $M_{\mathrm{d}} / M_{*}$ with SSFR holds over five orders of magnitude. This is consistent with the general idea that dust likely

\footnotetext{
${ }^{6}$ Note that the H-ATLAS stacks only extend down to $M_{*}=10^{8.5} \mathrm{M}_{\odot}$ so the drop in $M_{\mathrm{d}} / M_{*}$ for our low stellar mass sources does not contradict the statistically significant trend for the stacks.
}

traces the molecular ISM where star formation occurs (Dunne et al. 2000; Cortese et al. 2012a; Smith et al. 2012; Rowlands et al. 2014a). Despite the large differences in galaxy properties among the HIGH-high, HAPLESS and HRS samples, there is no evidence that they are forming stars in a fundamentally different way; they just have more or less star formation occurring as a result of their varying gas fractions. Fig. 4 (centre) also shows that, for all but the most immature sources in $\mathrm{HIGH}-\mathrm{low}$, dust mass is a reasonable indicator of SFR across a very wide range of $M_{*}$ and galaxy type.

Since $M_{\mathrm{H}_{\mathrm{I}}} / M_{*}$ is a proxy for how far a galaxy is through its evolution, the correlation seen in the right-hand panel of Fig. 4 implies that $M_{\mathrm{d}} / M_{*}$ depends on the evolutionary state. As galaxies evolve, they move from high to low $M_{\mathrm{H}_{\mathrm{I}}} / M_{*}$ and (for HIGH-high, the HRS and HAPLESS) $M_{\mathrm{d}} / M_{*}$ decreases. The unevolved sources in $\mathrm{HIGH}-$ low clearly lie below the relation for the other samples and imply a rising $M_{\mathrm{d}} / M_{*}$ at the earliest stages of evolution $\left(M_{\mathrm{H}_{\mathrm{I}}} / M_{*}>10^{0.5}\right)$. At high gas fractions, dust is not a good tracer of the $H$ icontent. These galaxies must be increasing their dust content at a faster fractional rate than their stellar content. The combined samples have allowed us to find a peak in the specific dust mass $\left(M_{\mathrm{d}} / M_{*}\right)$ in the local Universe occurring at a gas fraction of $\sim 75$ per cent and a stellar mass of $M_{*}=10^{8.5}$. HiGH-low is the first sample of galaxies that precedes this peak $M_{\mathrm{d}} / M_{*}$ in an evolutionary sequence.

\subsection{Dust enrichment relations}

We next look at the dust content of the ISM as a function of stellar mass, NUV $-r$ colour and $M_{\mathrm{H}_{\mathrm{I}}} / M_{*}$ (Fig. 5), where we find different scaling relations for $\mathrm{HIGH}-$ low. For $\mathrm{HIGH}$-high, HAPLESS and the $\mathrm{HRS}$, there is a weak but significant correlation between $M_{\mathrm{d}} / M_{\mathrm{H}}$ and $M_{*}(r=0.47)$. For HiGH-low, however, we find a steeper slope (Table 2) and a significantly smaller $M_{\mathrm{d}} / M_{\mathrm{H}_{\mathrm{I}}}$ than expected from extrapolating the relation for the other samples. We find tighter scaling relations with NUV $-r$ colour $(r=0.66)$ and gas richness ( $r=-0.86$ ) for HiGH-high, HAPLESS and the HRS, and again an offset towards lower dust enrichment for HIGH-low. Note again the offset between the HIGH-high/HAPLESS samples and the HRS for the stellar mass scaling relations (cf. Figs 3 and 4). Once again H I-deficient galaxies are offset when $M_{\mathrm{d}} / M_{\mathrm{H}_{\mathrm{I}}}$ is plotted against $M_{*}$, yet this offset disappears for the more fundamental relations of $M_{\mathrm{d}} / M_{\mathrm{HI}}$ with NUV $-r$ colour and $M_{\mathrm{H}_{\mathrm{I}}} / M_{*}$. The offset between the 

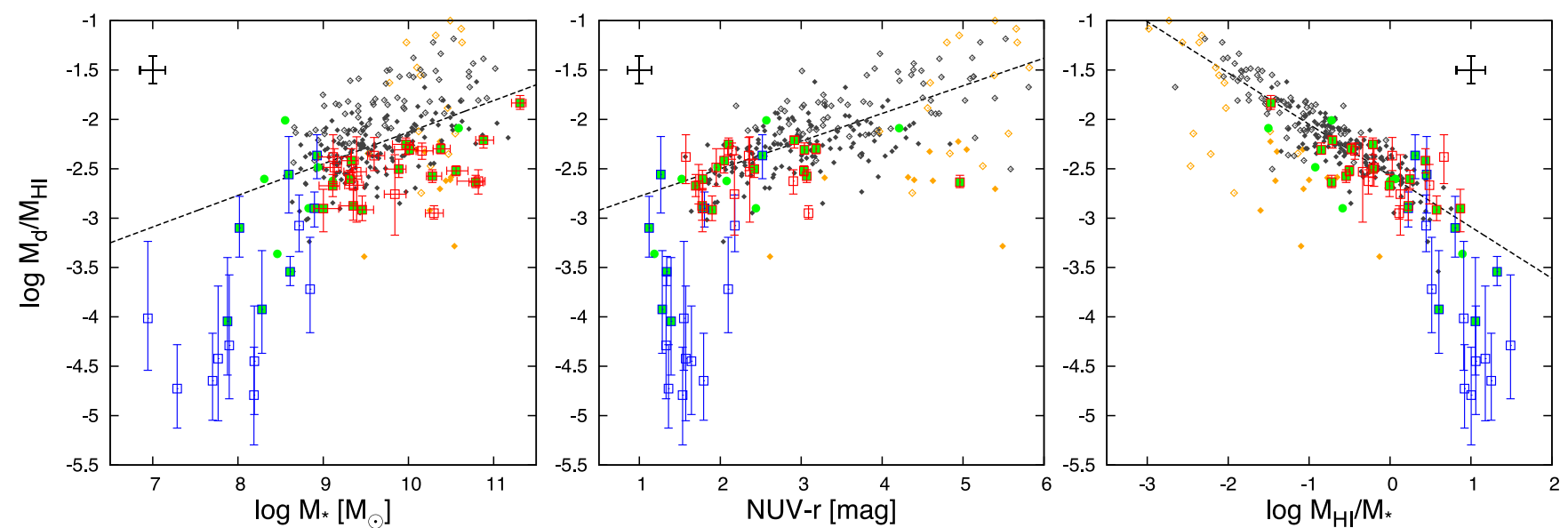

Figure 5. Dust enrichment relations for HRS, HAPLESS and HIGH. From left to right: scaling relations with stellar mass, NUV $-r$ colour (proxy for SSFR) and $M_{\mathrm{HI}} / M_{*}$. The line of best fit for the combined 'evolved' samples (HIGH-high, HAPLESS and HRS) is shown as a black dashed line. The HIGH-low sample lies significantly below the trend for the other samples and has a steeper slope. The symbols are the same as in Fig. 3.

samples is once again caused by differences in gas fractions at fixed stellar mass. Our interpretation of these dust enrichment relations is as follows:

Gas is continuously converted into stars, and dust is produced at the endpoints of stellar evolution (supernovae and asymptotic giant branch stars). Yet at the same time, dust is destroyed by shocks and also via astration as the ISM at the ambient dustto-gas ratio forms the next generation of stars. For HIGH-high, HAPLESS and the HRS, we have found positive correlations of the dust-to-gas ratio with stellar mass and NUV $-r$ colour, together with a negative correlation with the gas richness, showing that $M_{\mathrm{d}} / M_{\mathrm{H}}$ increases monotonically as galaxies move through their evolution. This implies that the dust destruction budget is not dominated by dust destruction through shocks or sputtering. If it was, we would observe a decrease in the dust-to-gas ratio as galaxies evolve. Some of the ETGs in HRS may be an exception to this. These ETGs are bright X-ray sources and some have AGNs in their centres. The hot gas in these sources erodes and breaks up the dust grains (sputtering), significantly reducing the dust mass and resulting in the outliers towards low $M_{\mathrm{d}} / M_{\mathrm{H}_{\mathrm{I}}}$ for HRS in Fig. 5.

In Fig. 6, we follow $\mathrm{C} 15$ in plotting $M_{\mathrm{d}} / M_{\text {bary }}$ mass ratio versus gas fraction $f_{\mathrm{g}}$ (their fig. 21) where we define the baryon mass as $M_{\text {bary }}=M_{\mathrm{H}_{\mathrm{I}}}+M_{*}$ and $f_{\mathrm{g}}=M_{\mathrm{H}_{\mathrm{I}}} /\left(M_{\mathrm{H}_{\mathrm{I}}}+M_{*}\right)$. Note that we do not have $\mathrm{CO}$ data for HIGH and HAPLESS so we cannot measure the molecular gas mass present in these galaxies. We also follow $\mathrm{C} 15$ in comparing the observations with a simple, closed box chemical evolution track ${ }^{7}$ showing the expected change in $M_{\mathrm{d}} / M_{\text {bary }}$ with gas fraction for a Milky Way type star formation history (Yin et al. 2009). The track (solid line; same as C15) shows the evolution of a galaxy as it evolves from gas-rich to gas-poor, with gas consumed by star formation.

Combining $\mathrm{HIGH}$ with HAPLESS and HRS allows us to sample a wider range of $f_{\mathrm{g}}$. As in C15, we see $M_{\mathrm{d}} / M_{\text {bary }}$ first rises steeply, then levels off and then drops again as galaxies evolve from high to low gas fractions. This supports the idea of the dust content being built up as galaxies move through the early stages of their evolution (gas fraction $>0.8$ ). The dust content then plateaus as dust destruction

\footnotetext{
${ }^{7}$ Further details on the model are presented in Rowlands et al. (2014b; see also Morgan \& Edmunds 2003).
}

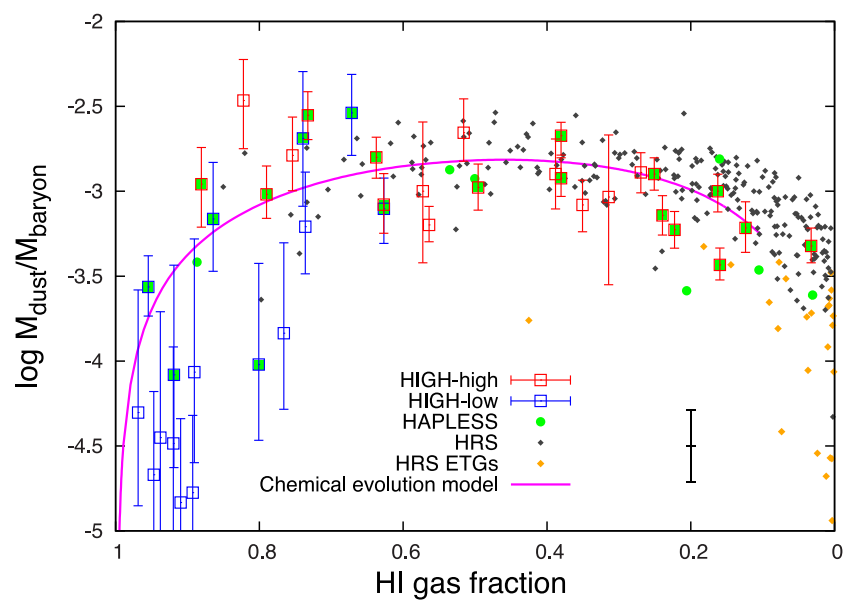

Figure 6. $M_{\mathrm{d}} / M_{\text {bary }}$ against gas fraction (without molecular hydrogen) reveals the evolution of dust. As galaxies evolve, the dust content first increases (high gas fraction), then reaches its peak for a gas fraction of $\sim 0.5$ and afterwards decreases as dust is consumed together with the gas (low gas fraction tail). A chemical evolution model (C15) is also shown.

through astration balances the dust production. Note that while the position of a galaxy in Fig. 6 does not depend on its total mass, since both axes are ratios, when sampling at the current epoch we find that the highest $M_{*}$ galaxies are at the right of the plot and the lowest $M_{*}$ are at the left, because massive galaxies go through their evolution faster. Including the $\mathrm{HIGH}$ sample provides crucial information at the highest gas fractions compared to the initial study in $\mathrm{C} 15$.

Of course, galaxies are far more complex than our simple model, with inflows, outflows and dust destruction expected to be important factors. A more detailed study of the build-up of dust at high gas fractions will be presented in paper II (De Vis et al., in preparation), and trends with metallicity will also be studied (high gas fraction sources have significantly lower metallicities than low gas fraction sources). However, even with the simplistic approach in $\mathrm{C} 15$, the model is still able to match the observed overall shape of the build-up and destruction of dust as a galaxy evolves. 

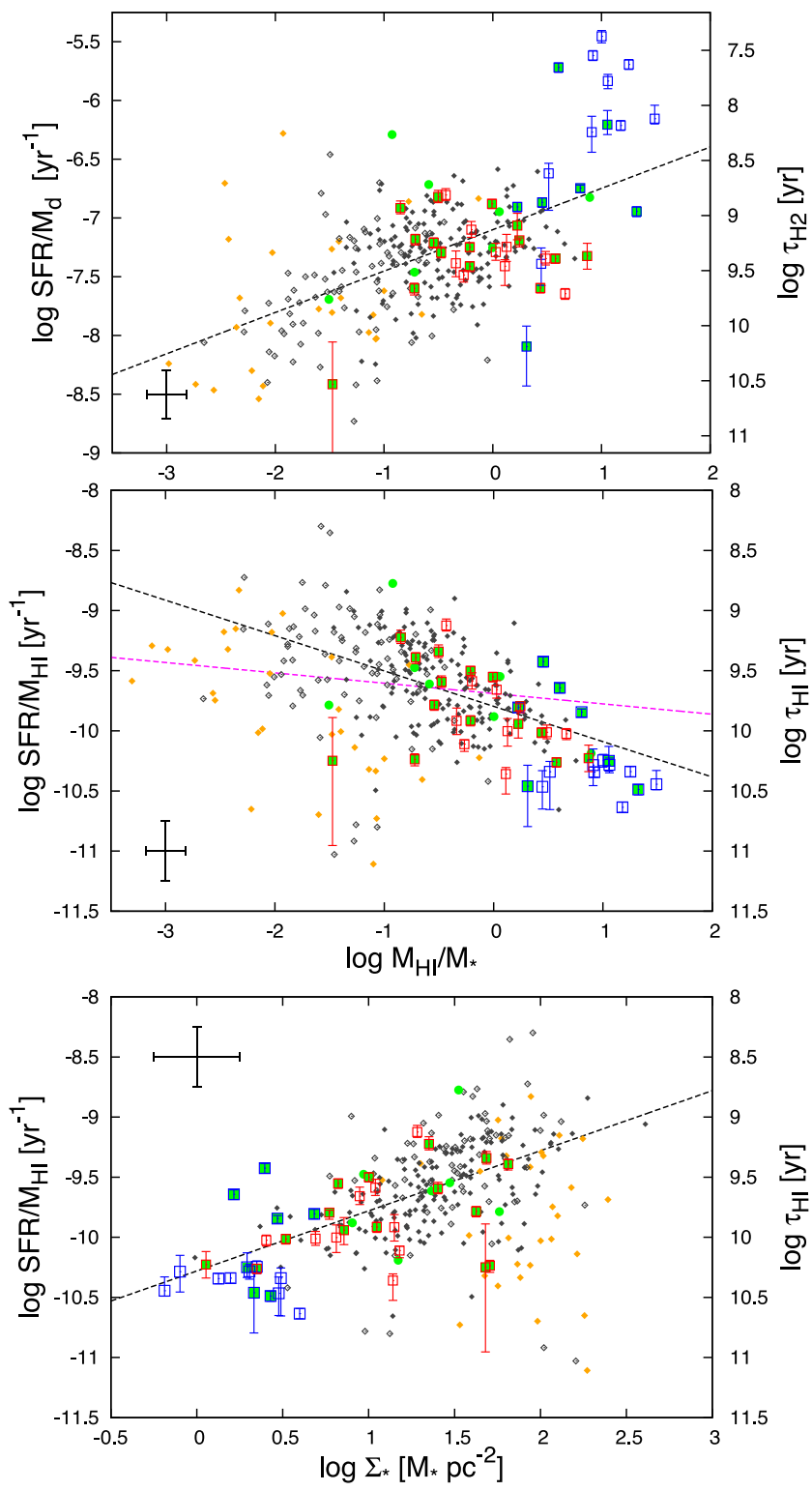

Figure 7. Top: SFR $/ M_{\mathrm{d}}$ against $M_{\mathrm{H}} / M_{*}$ showing a slow decline of $\mathrm{SFR} / M_{\mathrm{d}}$ as galaxies evolve. $\mathrm{HIGH}$-low is significantly offset towards higher $\mathrm{SFR} / M_{\mathrm{d}}$. The right axis shows the molecular gas depletion time-scale using $M_{\mathrm{d}}$ as a proxy. Centre: SFR $/ M_{\mathrm{H}_{\mathrm{I}}}$ against $M_{\mathrm{H}} / M_{*}$. There is a clear evolution towards higher SFR $/ M_{\mathrm{H}_{\mathrm{I}}}$ for more evolved sources (best-fitting line including all samples is shown by the dashed black line). The correlation resulting from a typical $\mathrm{H}$ I error of 0.1 dex has been determined using Monte Carlo (MC) simulations (dashed magenta line). Bottom: SFR per H I mass $\left(\mathrm{SFR} / M_{\mathrm{HI}}\right)$ against stellar mass surface density $\Sigma_{*}$.

\section{THE EVOLUTION OF STAR FORMATION EFFICIENCY}

In Fig. 7, we look at two measures of the star formation efficiency of the galaxies as a function of their gas fraction (or evolutionary status). In the top panel, we consider SFR $/ M_{\mathrm{d}}$, while in the bottom two panels we show SFR $/ M_{\mathrm{H}_{\mathrm{I}}}$. Our interpretation of these quantities is that $\mathrm{SFR} / M_{\mathrm{d}}$ is a proxy for the molecular star formation efficiency to the extent that dust is a good tracer of molecular gas in galaxies (Dunne et al. 2000; Planck Collaboration XIX 2011; Corbelli et al. 2012; Rowlands et al. 2014a; Santini et al. 2014; Scoville et al. 2014). As molecular gas is directly involved in star formation (e.g.
Bigiel et al. 2008; Schruba et al. 2011), SFR/ $M_{\mathrm{H}_{2}}\left(\right.$ aka SFR $\left./ M_{\mathrm{d}}\right)$ is an indicator of the efficiency with which gas is converted to stars inside the radius at which molecular clouds are present in the galaxy. ${ }^{8}$

On the other hand, atomic hydrogen does not directly form stars; it must first make a transition to molecular form. SFR $/ M_{\mathrm{H}_{\mathrm{I}}}$ is therefore not a true star formation efficiency but rather an indication of how effectively the $\mathrm{H}_{\mathrm{I}}$ is able to turn into the molecular form and subsequently forms stars. With this distinction in mind, we now turn to the trends shown in Fig. 7. Taken at face value, and assuming a canonical value for $M_{\mathrm{d}} / M_{\mathrm{H}_{2}}$ of 0.007 (Draine et al. 2007; see also Corbelli et al. 2012), the top panel of Fig. 7 shows that the star formation efficiency in galaxies declines as they evolve, with the relationship in Table 2 indicating a rise in the molecular gas depletion time-scale $\left(\tau_{\mathrm{H}_{2}}\right)$ from 1.7 to $4 \mathrm{Gyr}$ over a range in gas fraction from 80 to 10 per cent. The HIGH-low sample lies well above this relationship indicating either a much shorter molecular gas depletion time (average of $140 \mathrm{Myr}$ ) or a much lower $M_{\mathrm{d}} / M_{\mathrm{H}_{2}}$ ratio (by a factor of $\sim 10)$. Resolving this issue would require resolved $\mathrm{CO}+$ Hi maps for these sources.

Studies of the other main molecular gas tracer (CO) in local galaxies, selected over a range of stellar mass from $8.5<\log \mathrm{M}_{*}<$ 11.5 , find a similar result that the star formation efficiency increases (or the gas depletion time decreases) as the stellar mass decreases and as SSFR increases (Saintonge et al. 2011; Boselli et al. 2014a; Bothwell et al. 2014). ${ }^{9}$ These studies find a range of $\tau_{\mathrm{H}_{2}}$ from $100 \mathrm{Myr}$ to $5 \mathrm{Gyr}$ over the same range of stellar mass as sampled here, although our study contains three samples selected in very different ways (dust, gas and stellar content). ${ }^{10}$

In the centre panel of Fig. 7, we find that there is considerable evolution in SFR/ $M_{\mathrm{H}_{\mathrm{I}}}(r=-0.53)$ such that more evolved galaxies have higher star formation per $\mathrm{H}_{\mathrm{I}}$ mass (shorter $\mathrm{H}_{\mathrm{I}}$ depletion times, $\tau_{\mathrm{H}}$, assuming constant SFR and no re-supply of gas). We must be cautious in interpreting Fig. 7 (centre) as the quantity $\mathrm{M}_{\mathrm{H}}$ is present in both the $x$ and $y$ axes. Monte Carlo simulations were used to confirm that this relation cannot be due to biases introduced by the errors in $M_{\mathrm{H}_{\mathrm{I}}}$. For each source in the sample, we generated an artificial $\mathrm{H}_{\mathrm{I}}$ mass so that its $\mathrm{SFR} / M_{\mathrm{H}_{\mathrm{I}}}$ is equal to the average $\mathrm{SFR} / M_{\mathrm{H}_{\mathrm{I}}}$ in the whole sample (the null hypothesis is that there is no evolution in SFR $/ M_{\mathrm{H}_{\mathrm{I}}}$ ) and then added Gaussian scatter with a standard deviation of 0.1 dex (typical $M_{\mathrm{H}_{\mathrm{I}}}$ error). This process was repeated 100 times and the resulting average trend is shown by the magenta line in Fig. 7 (centre). The error on $\mathrm{M}_{\mathrm{H} 1}$ does introduce an artificial correlation; however, the observed slope in Fig. 7 (centre) is significantly steeper and we believe this is a real effect.

The galaxies with the highest gas fractions, which were previously found to be the most actively star-forming galaxies in terms of their stellar mass (SSFR) and their dust mass (SFR/ $M_{\mathrm{d}}$ ), are now least active with respect to their H I mass (they have the lowest $\mathrm{SFR} / M_{\mathrm{H}_{\mathrm{I}}}$ ). The $\mathrm{H}$ I depletion time-scales range from 0.2 to $63 \mathrm{Gyr}$,

\footnotetext{
${ }^{8}$ For a true measure of the efficiency of converting dense gas into stars within star-forming regions, it is necessary to choose a high-density molecular tracer (e.g. HCN; Gao \& Solomon 2004; Papadopoulos et al. 2012).

${ }^{9}$ Earlier studies of the molecular gas depletion times in local spiral galaxies found a constant $\tau_{\mathrm{H}_{2}}$ of $\sim 2 \mathrm{Gyr}$ (e.g. Bigiel et al. 2008; Leroy et al. 2008); however, these studies probed a much smaller range of intrinsic stellar masses or gas fractions and so are not thought to contradict the later findings of these larger studies.

${ }^{10}$ One of the samples (HRS) is the same as that used by Boselli et al. (2014a) though we are using dust as a tracer of $\mathrm{H}_{2}$ rather than $\mathrm{CO}$.
} 
with the most gas-rich $\left(M_{\mathrm{H}_{\mathrm{I}}}>\mathrm{M}_{*}\right)$ sources capable of sustaining their current SFRs for longer than the Hubble time. Previous studies find a comparable range in the value of $\tau_{\mathrm{H}_{\mathrm{I}}}$ but no trend with any of the parameters which correlate with $\tau_{\mathrm{H}_{2}}$, e.g. stellar mass, SSFR (Saintonge et al. 2011; Bothwell et al. 2014; Boselli et al. 2014a). Similarly, we do not find a correlation of $\tau_{\mathrm{H}}$ with either stellar mass or SSFR.

There is, however, a known relationship between $\tau_{\mathrm{H}_{\mathrm{I}}}$ and stellar mass surface density $\left(\Sigma_{*}\right)$ within galaxies. The THINGS survey (Leroy et al. 2008; Walter et al. 2008) found $\mathrm{SFR} / M_{\mathrm{H}_{\mathrm{I}}}$ to be a strong and almost linear function of stellar mass surface density in the outer regions of spirals and in dwarfs, where the ISM is dominated by H I. Within the inner regions of spiral galaxies, the higher stellar mass surface density produces a higher hydrostatic pressure in the ISM (Elmegreen 1989; Wong \& Blitz 2002) favouring the conversion of $\mathrm{H}_{\mathrm{I}}$ to $\mathrm{H}_{2}$ and results in a molecular-dominated region where the star formation efficiency $\left(\tau_{\mathrm{H}_{2}}\right)$ is constant. We find a correlation $(r=0.58)$ between the global $\tau_{\mathrm{H}_{\mathrm{I}}}$ and stellar mass surface density in the bottom panel of Fig. 7. This is the first time that such a relationship has been reported for global values between galaxies.

We can use Fig. 7 (bottom) to interpret the top two panels as being the result of an increasing efficiency of conversion of $\mathrm{HI}_{\mathrm{I}} \rightarrow \mathrm{H}_{2}$ as galaxies become more dominated by their stellar mass. As galaxies build up their stellar mass and increase in $\Sigma_{*}$, they create the conditions for $\mathrm{H}_{2}$ formation across a wider area; and their $\mathrm{H}_{\mathrm{I}}$ reservoirs are depleted due to conversion to $\mathrm{H}_{2}$ and thence to stars. As galaxies become very dominated by stars and have large bulges, they can be $\mathrm{H}_{2}$ dominated over large areas and their $\mathrm{H}_{\mathrm{I}}$ reservoirs will be relegated to the outskirts of the galaxy. In very evolved galaxies (e.g. ETGs), the presence of gas and star formation will be more strongly correlated with recent interactions or environmental effects (Davis et al. 2011; Kaviraj et al. 2012, 2013; Davies et al. 2015). This may explain the very large scatter in $\tau_{\mathrm{H}_{\mathrm{I}}}$ for the lowest gas fraction galaxies.

This general picture is not strongly dependent on an assumption of a constant dust-to- $\mathrm{H}_{2}$ ratio that ratio would need to vary by several orders of magnitude to invalidate this interpretation. Detailed observations of resolved $\mathrm{CO}, \mathrm{H}$ I and dust would be required to further elaborate on this.

\section{DUST HEATING IN THE DIFFUSE ISM}

Dust in the diffuse ISM is heated by the interstellar radiation field (ISRF), which has contributions from both old and young stellar populations. Dust in birth clouds experiences more intense and harsh radiation fields in the photo-dissociation regions. To account for this, MAGPHYS has a parameter, $f_{\mu}$, which represents the fraction of the total dust luminosity arising in the diffuse ISM. The majority of the dust mass resides in a cold $(10 \mathrm{~K}<T<30 \mathrm{~K})$ diffuse dust component, whereas in most actively star-forming galaxies a large fraction of the dust luminosity is due to a warm $(30 \mathrm{~K}<T<$ $60 \mathrm{~K}$ ) dust component arising in birth clouds (further details of the MAGPHYS components are found in Section 2.4).

In a typical galaxy in the local Universe, much of the stellar mass is in low-mass stars, yet the small fraction of massive, shortlived stars radiate much more strongly at UV wavelengths. This UV radiation is highly susceptible to absorption by dust and the highenergy UV photons can cause much of the dust heating (e.g. Draine et al. 2007) even though they only make up a very small fraction of the photons in the ISRF. In birth clouds, the UV photons from young stars dominate the dust heating, but even in the diffuse ISM

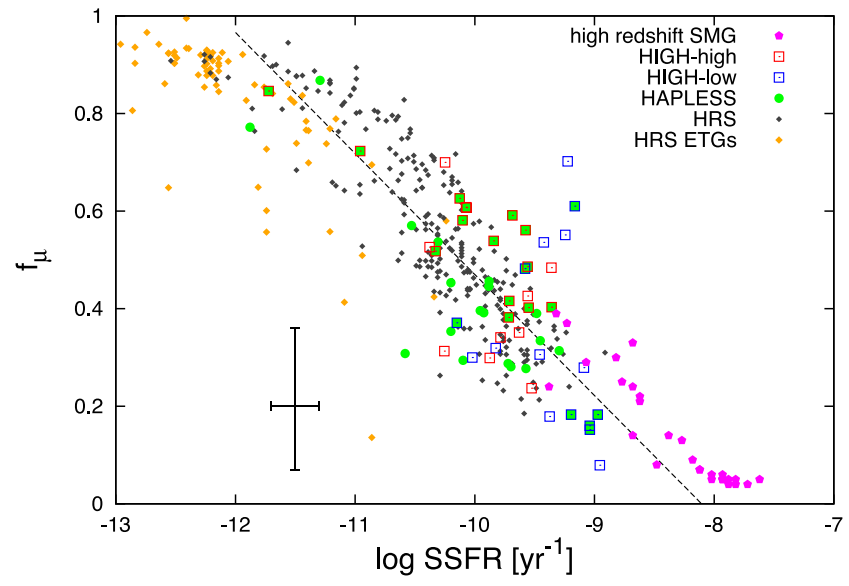

Figure 8. Influence of SSFR on the fraction of the total luminosity that originates in the diffuse ISM $\left(f_{\mu}\right)$. Less actively star-forming galaxies have a larger fraction of their dust luminosity originating in the diffuse ISM. The line of best fit for combined HAPLESS, HIGH and SMG (Rowlands et al. 2014a) samples is shown as a dashed black line.

the polycyclic aromatic hydrocarbons, small grains (stochastically heated and emitting at MIR) and warm dust components are still mostly heated by UV photons that leak from the birth clouds and form part of the diffuse ISRF (Devereux \& Young 1990; Kennicutt 1998; Calzetti et al. 2005, 2007; Boquien et al. 2010; Bendo et al. 2012; Kirkpatrick et al. 2014). Many literature works have studied the sources of dust heating for the bulk of the dust mass in the diffuse ISM, and found that both the young stars in star-forming regions and the diffuse evolved populations heat the diffuse dust (Bendo et al. 2010; Boselli et al. 2010b, 2012; Foyle et al. 2013; Ciesla et al. 2014; Cortese et al. 2014; Draine et al. 2014; Kirkpatrick et al. 2014; Bendo et al. 2015, C15). In this section, we will study which parameters drive change in $f_{\mu}$ and cold dust temperature, and investigate which source of dust heating dominates in a particular galaxy for a wide range of gas fractions.

Fig. 8 shows an anticorrelation of $f_{\mu}$ with SSFR for all the samples. In order to probe to the highest SSFR, we have included the high-redshift SMGs from Rowlands et al. (2014a). As expected, for most galaxies the fraction of the total dust luminosity originating in the birth clouds $\left(1-f_{\mu}\right)$ is proportional to the star-forming activity of the galaxy. This would be the case if a reasonable fraction of the energy in the birth clouds was being absorbed locally and re-radiated by dust (i.e. at least moderate $A_{\text {FUV }}$ ). Outliers can occur if the UV attenuation in the birth clouds is very low, allowing most of the UV energy to escape and heat the dust in the diffuse dust component. We indeed find that the outliers towards high $f_{\mu}$ in Fig. 8 are all amongst the least attenuated sources in the sample $\left(A_{\mathrm{FUV}}<0.35\right.$; see the following section). On the other hand, outliers can also occur if a considerable fraction of the dust is heated by AGN activity or the hot X-ray halo that is often present in ETGs, as these sources of heating are not included in the MAGPHYS prescription. All the outliers towards low $f_{\mu}$ are ETGs and the strongest outliers are known bright X-ray sources.

Both the young stars (traced by the SFR) and the old stars (traced by $M_{*}$ ) play a role in heating the diffuse dust. Fig. 9 provides us with a graphical way to understand the contributions from the old and young stellar populations to the total and cold dust heating. We have plotted $M_{*} / M_{\mathrm{d}}$ against $\mathrm{SFR} / M_{\mathrm{d}}$ and colour-coded the data by cold dust temperature and $f_{\mu}$, respectively (again including the SMGs from Rowlands et al. 2014a). 


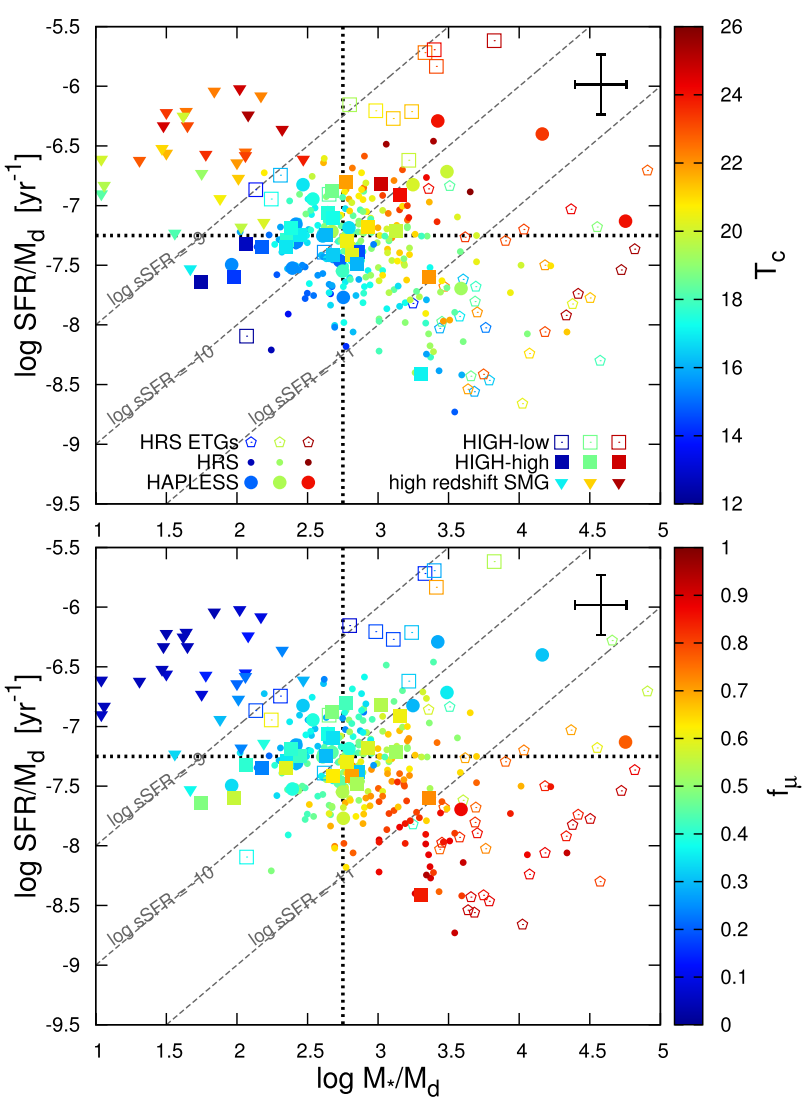

Figure 9. $M_{*} / M_{\mathrm{d}}$ against $\mathrm{SFR} / M_{\mathrm{d}}$, colour-coded by cold dust temperature $T_{\mathrm{c}}$ (top) and the fractional contribution of diffuse dust to the total dust luminosity, $f_{\mu}$ (bottom) in order to study the sources of dust heating. These plots have been divided into four 'heating' quadrants to highlight the differences. Lines of constant SSFR are shown in dashed grey lines.

Even discounting the SMGs, ${ }^{11}$ which generally lie off the main sequence of star formation, we find only a very weak correlation between SFR and $\mathrm{M}_{*}$ afternormalizing by dust mass. There is a clear trend towards higher temperatures as one goes to higher $M_{*} / M_{d}$ or $\mathrm{SFR} / M_{\mathrm{d}}$. For a fixed $M_{*} / M_{\mathrm{d}}$, we find the spread in temperatures largely follows the differences in SFR $/ M_{\mathrm{d}}$ and the same when fixing SFR $/ M_{\mathrm{d}}$ and varying $M_{*} / M_{\mathrm{d}}$. This explains why ETGs have warm $T_{\mathrm{c}}$, as, despite having low SFR, their $M_{*} / M_{\mathrm{d}}$ are the highest, and so their old stellar radiation fields are intense enough to heat the diffuse dust to warmer temperatures. At SFR $/ M_{\mathrm{d}}>10^{-6.5} \mathrm{yr}^{-1}$, there no longer seems to be any dependence of $T_{\mathrm{c}}$ on $\mathrm{M}_{*} / \mathrm{M}_{\mathrm{d}}$, probably because the dust heating is completely dominated by the young stellar population for these galaxies and the old stellar population has little effect. For galaxies with SFR $/ M_{\mathrm{d}}<10^{-6.5} \mathrm{yr}^{-1}$, both the young and old stellar populations heat the cold dust component, with some dominated by one and some by the other.

Fig. 9 (bottom) shows that the direction of increasing $f_{\mu}$ (also direction of increasing SSFR) is nearly orthogonal to the direction of increasing $T_{\mathrm{c}}$. This means that the cold dust temperature is more or less independent of the fraction of the total dust luminosity originating in the diffuse ISM (i.e. $T_{\mathrm{c}}$ is not affected by the SSFR). However, the fraction of the dust luminosity originating from heating by old stars is inversely proportional to SSFR (Boquien et al. 2016). Galaxies in the upper left quadrant of Fig. 9 have most of

${ }^{11}$ Including the SMGs leads to an even weaker correlation. the dust luminosity originating in birth clouds and the dust heating is dominated by photons from young stars. Galaxies with moderate SSFR have dust emission from both birth clouds and the diffuse ISM as well as significant contributions to the heating of the diffuse dust from both young and old stars. These galaxies can be dust-rich and cold (lower left quadrant) or dust-poor and warmer (upper right quadrant). Finally, quiescent galaxies (lower right quadrant) have most of their dust luminosity originating in the diffuse ISM and this is heated mostly by the old stars.

\section{OBSCURATION}

To study how the UV obscuration depends on other galaxy properties, we have plotted the MAGPHYS $A_{\mathrm{FUV}}$ parameter against $M_{\mathrm{H}_{\mathrm{I}}} / M_{*}$, $\Sigma_{*}$ and dust mass in Fig. 10. In the left-hand panel, we find an anticorrelation $(r=-0.54)$ for $A_{\mathrm{FUV}}$ with $M_{\mathrm{H}_{\mathrm{I}}} / M_{*}$. As galaxies move through their evolution, from gas-rich to gas-poor (right to left on this plot) the obscuration initially increases. This makes sense as dust is continuously produced and mixed with the ISM. Note that all galaxies with $\log \mathrm{M}_{\mathrm{H}_{\mathrm{I}}} / \mathrm{M}_{*}>0.5$ approach $A_{\mathrm{FUV}}=0$, which corresponds to the limit of no obscuration. From Fig. 5, this corresponds to $\log M_{\mathrm{d}} / M_{\mathrm{H}}<-3$.

The sources with the highest obscuration have the highest cold dust temperatures and are, on average, slightly more inclined than the less obscured galaxies at the same $M_{\mathrm{H}_{\mathrm{I}}} / M_{*}$. In the latest stages of evolution, the obscuration decreases again as most of the dust mass is consumed due to astration (Fig. 6) or removed.

The large scatter in $A_{\mathrm{FUV}}$ at lower gas fractions is at least partly due to differences in the intrinsic stellar and dust geometries and inclinations of these galaxies. Attenuation strongly depends on how much of the dust is mixed into the diffuse ISM as opposed to being distributed in a more clumpy geometry, and on other geometric differences like scaleheights and scalelengths of the stellar and dust discs (Baes \& Dejonghe 2001; Bianchi 2008; Popescu et al. 2011; Holwerda et al. 2012). Investigating whether or not the star-dust geometry is the main factor that drives this scatter is a difficult task. One potential way to do that is by including realistic recipes for dust attenuation in hydrodynamical models of galaxy evolution, and comparing the attenuation properties of simulated mock galaxies to observed data. As both cosmological hydrodynamical simulations (e.g. Vogelsberger et al. 2014; Schaye et al. 2015) and $3 \mathrm{D}$ dust radiative transfer techniques (Steinacker, Baes \& Gordon 2013) have reached a level of maturity, this combination has recently become possible (e.g. Camps et al. 2016; Trayford et al., in preparation). Such an investigation is beyond the scope of this paper, but will be the subject of future work.

Grootes et al. (2013) found a relationship between the optical depth and stellar mass surface density $\Sigma_{*}$ of nearby spiral galaxies. We find a similar relationship ( $r=0.53$ ) when we plot $A_{\mathrm{FUV}}$ against $\Sigma_{*}$ in Fig. 10 (centre). The increased stellar mass potential associated with higher $\Sigma_{*}$ creates instabilities in the cold ISM, which leads to the formation of a thin dust disc (Dalcanton, Yoachim $\&$ Bernstein 2004). This changes the relative geometries of dust and stars, which provides a possible explanation for the changes in obscuration.

The attenuation by dust is expected to depend on the total column of dust along a photon's trajectory. We show how $A_{\mathrm{FUV}}$ varies with the total dust mass, ${ }^{12}$ colour-coded by gas fraction, in the right-hand

\footnotetext{
${ }^{12}$ Plotting $A_{\mathrm{FUV}}$ against the dust surface density (which could be argued to be a better tracer of the dust mass along a photon's path) does not change the results in any way.
} 

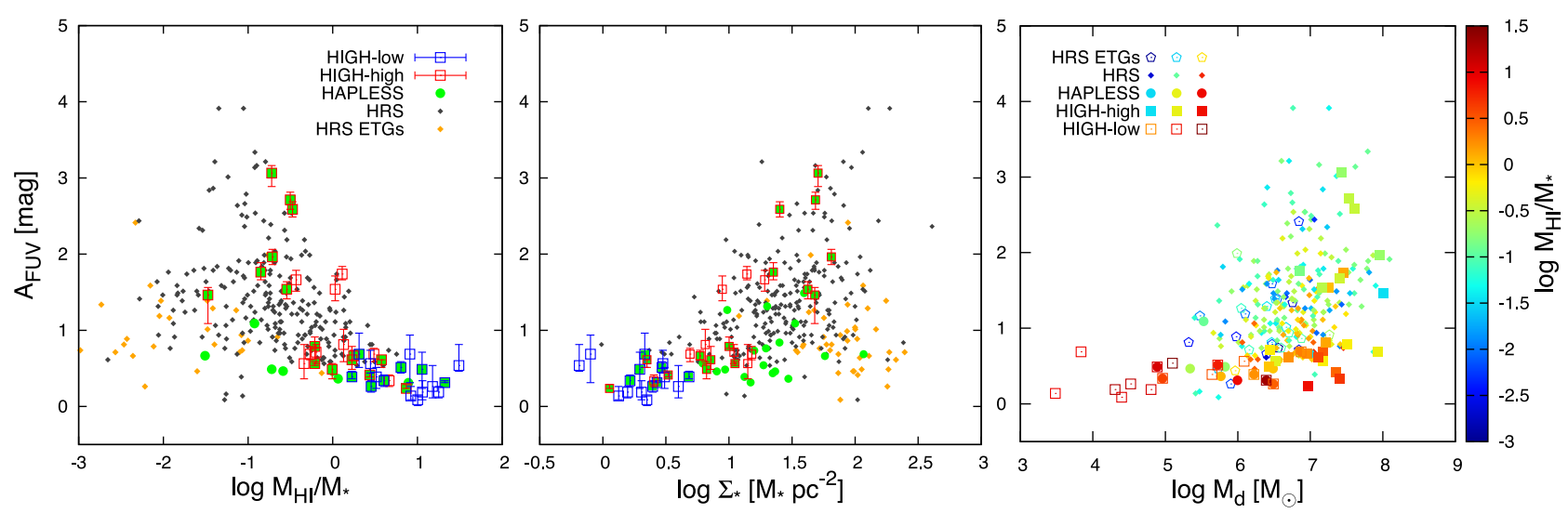

Figure 10. The variation of FUV attenuation $A_{\mathrm{FUV}}$ with galaxy parameters. Left: the obscuration increases as one moves from high to low gas fractions (i.e. from less to more evolved sources). Centre: relationship between $A_{\mathrm{FUV}}$ and stellar mass surface density $\Sigma_{*}$. Right: for low dust masses $\left(M_{\mathrm{d}}<10^{5} \mathrm{M}_{\odot}\right)$ there is little to no obscuration. We find a positive correlation between the obscuration and the dust mass above this value for the HAPLESS and HIGH samples. The outliers towards high obscuration at moderate dust masses are evolved sources in HRS $\left(\log M_{\mathrm{H}_{\mathrm{I}}} / M_{*}<-0.6\right)$. For all three plots, there is a large amount of scatter that correspond to a wide range of obscuration for a given $M_{\mathrm{d}}$ (likely due to different dust and stellar geometries).

panel of Fig. 10. We find a positive correlation $(r=0.38)$ but the relationship is not a simple power law, and there is a lot of scatter. At $M_{\mathrm{d}}<10^{5.5}$ the obscuration tends to zero, while at higher dust masses, there is a large range in obscuration and again this is likely due to different stellar and dust geometries or different extinction laws. In summary, we find no clear and simple link between UV obscuration and global galaxy properties.

\section{CONCLUSIONS}

We have studied the interplay of dust, gas and star formation for combined samples made up of local H I-, dust- and stellar-massselected galaxies, using MAGPHYS to determine physical properties from UV to submm photometry. The combined samples cover a wide range of gas fractions (proxy for the evolutionary state of a galaxy). Our main results are as follows:

(i) We have identified a subsample of $\mathrm{H}$ I-selected sources (HiGH-low) with very high gas fractions $\left(f_{\mathrm{g}}>80\right.$ per cent) and low stellar masses $\left(M_{*}<10^{9} \mathrm{M}_{\odot}\right)$. These probe the earliest stages of evolution, and have a much smaller dust content than expected from extrapolating published scaling relations for more evolved sources.

(ii) In the earliest stages of evolution $\left(f_{\mathrm{g}}>80\right.$ per cent), dust is not a good tracer of the gas content. The dust content relative to stellar mass first rises steeply with decreasing gas fraction, reaches a peak at a gas fraction of $\sim 75$ per cent (which for local galaxies is equivalent to a stellar mass of $\sim 10^{8.5} \mathrm{M}_{\odot}$ ) and then decreases together with gas fraction.

(iii) The galaxies with the highest gas fractions are the most actively star-forming galaxies relative to their stellar masses (SSFR) and relative to their $\mathrm{H}_{2}$ content (using dust as a proxy for $\mathrm{H}_{2}$ ).

(iv) We find a trend of decreasing $\mathrm{H}_{\mathrm{I}}$ depletion time with decreasing gas fraction such that the most gas-rich galaxies have the longest $\tau_{\mathrm{H}}$. We interpret this, together with the opposing behaviour of $\tau_{\mathrm{H}_{2}}$, as being due to the increasing efficiency with which $\mathrm{H}_{\mathrm{I}}$ can be converted to $\mathrm{H}_{2}$ as galaxies increase in stellar mass surface density with decreasing gas fraction.

(v) We confirm literature results that both old and young stellar populations play an important role in heating the diffuse dust component, and both can be the dominant contributor in individual systems. The SSFR determines which dominates. (vi) The FUV obscuration of galaxies shows no clear and simple link with global galaxy properties. Galaxies start out barely obscured and increase in obscuration as they evolve until the dust mass decreases significantly in the latest stages of evolution.

The derived scaling relations for the combined samples in this work span a wider range in gas fraction than previous relations in the literature, yet admittedly have somewhat complex selection biases. Since the sample size of the stellar-mass-selected sample (HRS) is eight times larger than the $\mathrm{HI}_{\mathrm{I}}$ and dust-selected samples, the scaling relations are therefore heavily weighted towards this sample. This especially affects the scaling relations with stellar mass, which show significant offsets between the differently selected samples. However, using the combined sample including the high gas fraction sources, we show that the most robust scaling relations for gas and dust are those linked to NUV $-r$ (SSFR) and gas fraction. These are tight relations which do not depend on sample selection or environment and are thus not affected by the complex selection criteria of the combined sample.

\section{ACKNOWLEDGEMENTS}

We thank the anonymous referee for useful suggestions. The authors gratefully acknowledge Martha Haynes, Riccardo Giovanelli and the ALFALFA team for supplying the latest ALFALFA survey data. The authors also thank Ariadna Manilla Robles, Asantha Cooray, Paul van der Werf and Michal Michalowski for helpful discussions. LD, SJM and RJI acknowledge support from the ERC in the form of the Advanced Investigator Program, COSMICISM. HLG, LD and SJM acknowledge support from the European Research Council in the form of Consolidator Grant CosmicDust. CJRC acknowledges support from the European Research Council (ERC) FP7 project DustPedia (PI: Jon Davies) and the STFC Doctoral Training Grant scheme. The H-ATLAS is a project with Herschel, which is an ESA space observatory with science instruments provided by European-led Principal Investigator consortia and with important participation from NASA. The H-ATLAS website is http://www.h-atlas.org/. GAMA is a joint European-SDSS DR7Australasian project based around a spectroscopic campaign using the Anglo-Australian Telescope. The GAMA input catalogue is based on data taken from the Sloan Digital Sky Survey and the UKIRT Infrared Deep Sky Survey. Complementary imaging 
of the GAMA regions is being obtained by a number of independent survey programs including GALEX MIS, VST KIDS, VISTA VIKING, WISE, Herschel-ATLAS, GMRT and ASKAP, providing UV to radio coverage. GAMA is funded by the STFC (UK), the ARC (Australia), the AAO and the participating institute. The GAMA website is: http://www.gama-survey.org/. This research has made use of Astropy, ${ }^{13}$ a community-developed core Python package for Astronomy (Astropy Collaboration 2013). This research has made use of TOPCAT ${ }^{14}$ (Taylor 2005), which was initially developed under the UK Starlink project, and has since been supported by PPARC, the VOTech project, the AstroGrid project, the AIDA project, the STFC, the GAVO project, the European Space Agency and the GENIUS project. This research has made use of APLpy, ${ }^{15}$ an open-source astronomical image plotting package for Python. This research has made use of NumPy ${ }^{16}$ (Walt, Colbert \& Varoquaux 2011), $\mathrm{SciPy}^{17}$ and MatPlotLib ${ }^{18}$ (Hunter 2007). This research has made use of the SIMBAD ${ }^{19}$ data base (Wenger et al. 2000) and the VizieR ${ }^{20}$ catalogue access tool (Ochsenbein, Bauer \& Marcout 2000), both operated at CDS, Strasbourg, France. This research has made use of SAOImage DS $9,{ }^{21}$ developed by the Smithsonian Astrophysical Observatory with support from the Chandra X-ray Science Center (CXC), the High Energy Astrophysics Science Archive Center (HEASARC) and the JWST Mission office at the Space Telescope Science Institute (STSI). This research has made use of the NASA/IPAC Extragalactic Database (NED, ${ }^{22}$ operated by the Jet Propulsion Laboratory, California Institute of Technology, under contract with the National Aeronautics and Space Administration.

\section{REFERENCES}

Abazajian K. N. et al., 2009, ApJS, 182, 543

Adelman-McCarthy J. K. et al., 2008, ApJS, 175, 297

Ahn C. P. et al., 2012, ApJS, 203, 21

Akritas M. G., Bershady M. A., 1996, ApJ, 470, 706

Astropy Collaboration, 2013, A\&A, 558, A33

Baes M., Dejonghe H., 2001, MNRAS, 326, 733

Baldry I. K. et al., 2012, MNRAS, 421, 621

Barnes D. G. et al., 2001, MNRAS, 322, 486

Bendo G. J. et al., 2010, A\&A, 518, L65

Bendo G. J. et al., 2012, MNRAS, 419, 1833

Bendo G. J. et al., 2015, MNRAS, 448, 135

Bertin E., Arnouts S., 1996, A\&AS, 117, 393

Bianchi S., 2008, A\&A, 490, 461

Bigiel F., Leroy A., Walter F., Brinks E., de Blok W. J. G., Madore B.,

Thornley M. D., 2008, AJ, 136, 2846

Bigiel F. et al., 2011, ApJ, 730, L13

Boquien M. et al., 2010, A\&A, 518, L70

Boquien M. et al., 2016, A\&A, 591, A6

Boselli A., Gavazzi G., Donas J., Scodeggio M., 2001, AJ, 121, 753

Boselli A. et al., 2010a, PASP, 122, 261

Boselli A. et al., 2010b, A\&A, 518, L61

Boselli A. et al., 2012, A\&A, 540, A54

\footnotetext{
${ }^{13} \mathrm{http} / / / \mathrm{www}$.astropy.org/

${ }^{14} \mathrm{http}: / /$ www.star.bris.ac.uk/mbt/topcat/

${ }^{15} \mathrm{http} / / /$ aplpy.github.io/

${ }^{16} \mathrm{http}: / /$ www.numpy.org/

${ }^{17} \mathrm{http}: / /$ www.scipy.org/

${ }^{18} \mathrm{http}: / /$ matplotlib.org/

${ }^{19} \mathrm{http}: / /$ simbad.u-strasbg.fr/simbad/

${ }^{20} \mathrm{http} / / /$ vizier.u-strasbg.fr/viz-bin/VizieR

${ }^{21} \mathrm{http}: / / \mathrm{ds} 9$. si.edu/site/Home.html

$22 \mathrm{http} / / /$ ned.ipac.caltech.edu/
}

Boselli A., Hughes T. M., Cortese L., Gavazzi G., Buat V., 2013, A\&A, 550, A114

Boselli A., Cortese L., Boquien M., 2014a, A\&A, 564, A65

Boselli A., Cortese L., Boquien M., Boissier S., Catinella B., Lagos C., Saintonge A., 2014b, A\&A, 564, A66

Boselli A., Fossati M., Gavazzi G., Ciesla L., Buat V., Boissier S., Hughes T. M., 2015, A\&A, 579, A102

Bothwell M. S. et al., 2014, MNRAS, 445, 2599

Bourne N. et al., 2012, MNRAS, 421, 3027

Bourne N. et al., 2013, MNRAS, 436, 479

Bourne N. et al., 2016, MNRAS, 462, 1714

Calzetti D. et al., 2005, ApJ, 633, 871

Calzetti D. et al., 2007, ApJ, 666, 870

Camps P., Trayford J. W., Baes M., Theuns T., Schaller M., Schaye J., 2016, MNRAS, 462, 1057

Catinella B. et al., 2010, MNRAS, 403, 683

Catinella B. et al., 2013, MNRAS, 436, 34

Ciesla L. et al., 2014, A\&A, 565, A128

Clark C. J. R. et al., 2015, MNRAS, 452, 397

Cluver M. E. et al., 2014, ApJ, 782, 90

Corbelli E. et al., 2012, A\&A, 542, A32

Cortese L., Catinella B., Boissier S., Boselli A., Heinis S., 2011, MNRAS, 415, 1797

Cortese L. et al., 2012a, A\&A, 540, A52

Cortese L. et al., 2012b, A\&A, 544, A101

Cortese L. et al., 2014, MNRAS, 440, 942

Cowie L. L., Songaila A., Hu E. M., Cohen J. G., 1996, AJ, 112, 839

da Cunha E., Charlot S., Elbaz D., 2008, MNRAS, 388, 1595

da Cunha E., Eminian C., Charlot S., Blaizot J., 2010, MNRAS, 403, 1894

Dalcanton J. J., Yoachim P., Bernstein R. A., 2004, ApJ, 608, 189

Davies L. J. M. et al., 2015, MNRAS, 452, 616

Davis T. A. et al., 2011, MNRAS, 417, 882

De Lucia G., Springel V., White S. D. M., Croton D., Kauffmann G., 2006 , MNRAS, 366, 499

Devereux N. A., Young J. S., 1990, ApJ, 350, L25

Draine B. T. et al., 2007, ApJ, 663, 866

Draine B. T. et al., 2014, ApJ, 780, 172

Driver S. P., Allen P. D., Liske J., Graham A. W., 2007, ApJ, 657, L85

Driver S. P. et al., 2009, Astron. Geophys., 50, 050000

Driver S. P. et al., 2011, MNRAS, 413, 971

Driver S. P. et al., 2016, MNRAS, 455, 3911

Dunne L., Eales S. A., 2001, MNRAS, 327, 697

Dunne L., Eales S., Edmunds M., Ivison R., Alexander P., Clements D. L., 2000, MNRAS, 315, 115

Dunne L. et al., 2011, MNRAS, 417, 1510

Eales S. et al., 2010, PASP, 122, 499

Elmegreen B. G., 1989, ApJ, 338, 178

Fisher J. R., Tully R. B., 1981, ApJS, 47, 139

Fixsen D. J., Cheng E. S., Gales J. M., Mather J. C., Shafer R. A., Wright E. L., 1996, ApJ, 473, 576

Foyle K. et al., 2013, MNRAS, 432, 2182

Gao Y., Solomon P. M., 2004, ApJ, 606, 271

Gavazzi G., Scodeggio M., 1996, A\&A, 312, L29

Gavazzi G., Fumagalli M., Fossati M., Galardo V., Grossetti F., Boselli A., Giovanelli R., Haynes M. P., 2013, A\&A, 553, A89

Giovanelli R. et al., 2005, AJ, 130, 2598

Griffin M. J. et al., 2010, A\&A, 518, L3

Grootes M. W. et al., 2013, ApJ, 766, 59

Haynes M. P. et al., 2011, AJ, 142, 170

Holland W. S. et al., 1999, MNRAS, 303, 659

Holwerda B. W. et al., 2012, A\&A, 541, L5

Hopkins A. M. et al., 2013, MNRAS, 430, 2047

Huang S., Haynes M. P., Giovanelli R., Brinchmann J., 2012, ApJ, 756, 113

Hunter J. D., 2007, Comput. Sci. Eng., 9, 90

Irwin M., 2010, UKIRT Newsletter, 26, 14

Issa M. R., MacLaren I., Wolfendale A. W., 1990, A\&A, 236, 237

James A., Dunne L., Eales S., Edmunds M. G., 2002, MNRAS, 335, 753

Kaviraj S. et al., 2012, MNRAS, 423, 49 
Kaviraj S. et al., 2013, MNRAS, 435, 1463

Kennicutt R. C., Jr, 1998, ApJ, 498, 541

Kessler M. F. et al., 1996, A\&A, 315, L27

Kirkpatrick A. et al., 2014, ApJ, 789, 130

Kreysa E. et al., 1998, in Phillips T. G., ed., Proc. SPIE Conf. Ser. Vol. 3357, Advanced Technology MMW, Radio, and Terahertz Telescopes. SPIE, Bellingham, p. 319

Kreysa E. et al., 2003, in Phillips T. G., Zmuidzinas J., eds, Proc. SPIE Conf. Ser. Vol. 4855, Millimeter and Submillimeter Detectors for Astronomy. SPIE, Bellingham, p. 41

Leroy A. K., Walter F., Brinks E., Bigiel F., de Blok W. J. G., Madore B., Thornley M. D., 2008, AJ, 136, 2782

Lisenfeld U., Ferrara A., 1998, ApJ, 496, 145

Lisenfeld U. et al., 2007, A\&A, 462, 507

Liske J. et al., 2015, MNRAS, 452, 2087

Meyer M. J. et al., 2004, MNRAS, 350, 1195

Morgan H. L., Edmunds M. G., 2003, MNRAS, 343, 427

Morrissey P. et al., 2007, ApJS, 173, 682

Neugebauer G. et al., 1984, ApJ, 278, L1

Ochsenbein F., Bauer P., Marcout J., 2000, A\&AS, 143, 23

Papadopoulos P. P., van der Werf P. P., Xilouris E. M., Isaak K. G., Gao Y., Mühle S., 2012, MNRAS, 426, 2601

Pilbratt G. L. et al., 2010, A\&A, 518, L1

Planck Collaboration I, 2014, A\&A, 571, A1

Planck Collaboration XIX, 2011, A\&A, 536, A19

Poglitsch A. et al., 2010, A\&A, 518, L2

Popescu C. C., Tuffs R. J., Dopita M. A., Fischera J., Kylafis N. D., Madore B. F., 2011, A\&A, 527, A109

Popping A., Braun R., 2011, A\&A, 527, A90

Richter O.-G., Tammann G. A., Huchtmeier W. K., 1987, A\&A, 171, 33

Riess A. G. et al., 2011, ApJ, 730, 119

Roussel H., 2013, PASP, 125, 1126

Rowlands K. et al., 2014a, MNRAS, 441, 1017

Rowlands K., Gomez H. L., Dunne L., Aragón-Salamanca A., Dye S., Maddox S., da Cunha E., Werf P. v. d., 2014b, MNRAS, 441, 1040

Saintonge A. et al., 2011, MNRAS, 415, 32

Sanders D. B., Scoville N. Z., Soifer B. T., 1991, ApJ, 370, 158

Sanders D. B., Mazzarella J. M., Kim D.-C., Surace J. A., Soifer B. T., 2003, AJ, 126, 1607

Sandstrom K. M. et al., 2013, ApJ, 777, 5

Santini P. et al., 2014, A\&A, 562, A30

Schaye J. et al., 2015, MNRAS, 446, 521

Schiminovich D. et al., 2007, ApJS, 173, 315

Schneider S. E., Helou G., Salpeter E. E., Terzian Y., 1986, AJ, 92, 742

Schruba A. et al., 2011, AJ, 142, 37

Scoville N. et al., 2014, ApJ, 783, 84

Seibert M. et al., 2012, in American Astronomical Society Meeting Abstracts \#219. p. 340.01

Smith D. J. B. et al., 2011, MNRAS, 416, 857

Smith D. J. B. et al., 2012, MNRAS, 427, 703

Steinacker J., Baes M., Gordon K. D., 2013, ARA\&A, 51, 63

Sulentic J. W., Arp H., 1983, AJ, 88, 489

Sutherland W., 2012, Science from the Next Generation Imaging and Spectroscopic Surveys

Taylor M. B., 2005, in Shopbell P., Britton M., Ebert R., eds, ASP Conf. Ser. Vol. 347, Astronomical Data Analysis Software and Systems XIV. Astron. Soc. Pac., San Francisco, p. 29

Taylor C. L., Brinks E., Grashuis R. M., Skillman E. D., 1995, ApJS, 99, 427

Tonry J. L., Blakeslee J. P., Ajhar E. A., Dressler A., 2000, ApJ, 530, 625

Valiante E. et al., 2016, MNRAS, 462, 3146

Verley S. et al., 2010, A\&A, 518, L68

Viaene S. et al., 2014, A\&A, 567, A71

Viaene S. et al., 2016, A\&A, 586, A13
Vogelsberger M. et al., 2014, MNRAS, 444, 1518

Walt S. v. d., Colbert S. C., Varoquaux G., 2011, Comput. Sci. Eng., 13, 22

Walter F., Brinks E., de Blok W. J. G., Bigiel F., Kennicutt R. C., Jr, Thornley M. D., Leroy A., 2008, AJ, 136, 2563

Wenger M. et al., 2000, A\&AS, 143, 9

Werner M. W. et al., 2004, ApJS, 154, 1

Wong T., Blitz L., 2002, ApJ, 569, 157

Wong O. I. et al., 2006, MNRAS, 371, 1855

Wright E. L. et al., 2010, AJ, 140, 1868

Yin J., Hou J. L., Prantzos N., Boissier S., Chang R. X., Shen S. Y., Zhang B., 2009, A\&A, 505, 497

Zhukovska S., 2014, A\&A, 562, A76

Zwaan M. A. et al., 2004, MNRAS, 350, 1210

\section{APPENDIX A: EXCEPTIONS TO THE APERTURE PHOTOMETRY}

\section{A1 IRAS SCANPI photometry}

We used the Scan Processing and Integration Tool (SCANPI ${ }^{23}$ ), following the procedure of Sanders et al. (2003) to measure $60 \mu \mathrm{m}$ fluxes and uncertainties for our sources. For a third of the sample, no reliable detection could be found at the location of the source. For these sources, the scans were inspected manually and an upper limit was defined for the flux as three times the local rms.

\section{A2 Herschel PACS photometry}

We dealt with PACS photometry as described in C15, using apertures based on the $250 \mu \mathrm{m}$ source size. In contrast to $\mathrm{C} 15$, we use the H-ATLAS DR1 nebulized ${ }^{24}$ maps for all our sources. The filtering applied to the maps could lead to a localized negative background for very extended sources (Valiante et al. 2016). By limiting the PACS aperture to the obvious extent of the dust emission we are minimizing the effects of these large-scale background issues and increasing the accuracy and reliability of the flux measurements. Where we do not have a strong enough $250 \mu \mathrm{m}$ detection to reliably determine an aperture, we use an aperture 0.8 times the largest aperture size from the other bands. This factor was determined to be the average ratio of $r_{\mathrm{ap}(250)} / r_{\mathrm{ap}(\max )}$ for sources with $\mathrm{SNR}_{250}>$ 5 within the aperture. We have performed tests that there are no significant systematic differences in the fluxes obtained when either using a larger aperture or when the raw SCANAmORPHOS (Roussel 2013) maps are used instead.

\section{APPENDIX B: PROPERTIES OF THE HIGH GALAXIES}

Basic properties for our $\mathrm{H}$ I-selected sample, such as identifiers, positions and sizes, are given in Table B1. The H I fluxes, H I masses, references and other $\mathrm{HI}$-derived properties are given in Table B2. The UV to FIR photometry for the $\mathrm{H}_{\mathrm{I}}$-selected $\mathrm{HIGH}$ sample can be found in Table B3 . Multiwavelength imagery of the HIGH galaxies is shown in Fig. B1. The MAGPHYs fits to the spectral energy distributions of the HIGH sources are shown in Fig. B2.

\footnotetext{
${ }^{23}$ Provided by the NASA/IPAC Infrared Science Archive: http://irsa.ipac.caltech.edu/applications/Scanpi/.

${ }^{24}$ NEBULISER is an algorithm to remove background emission (Irwin 2010).
} 


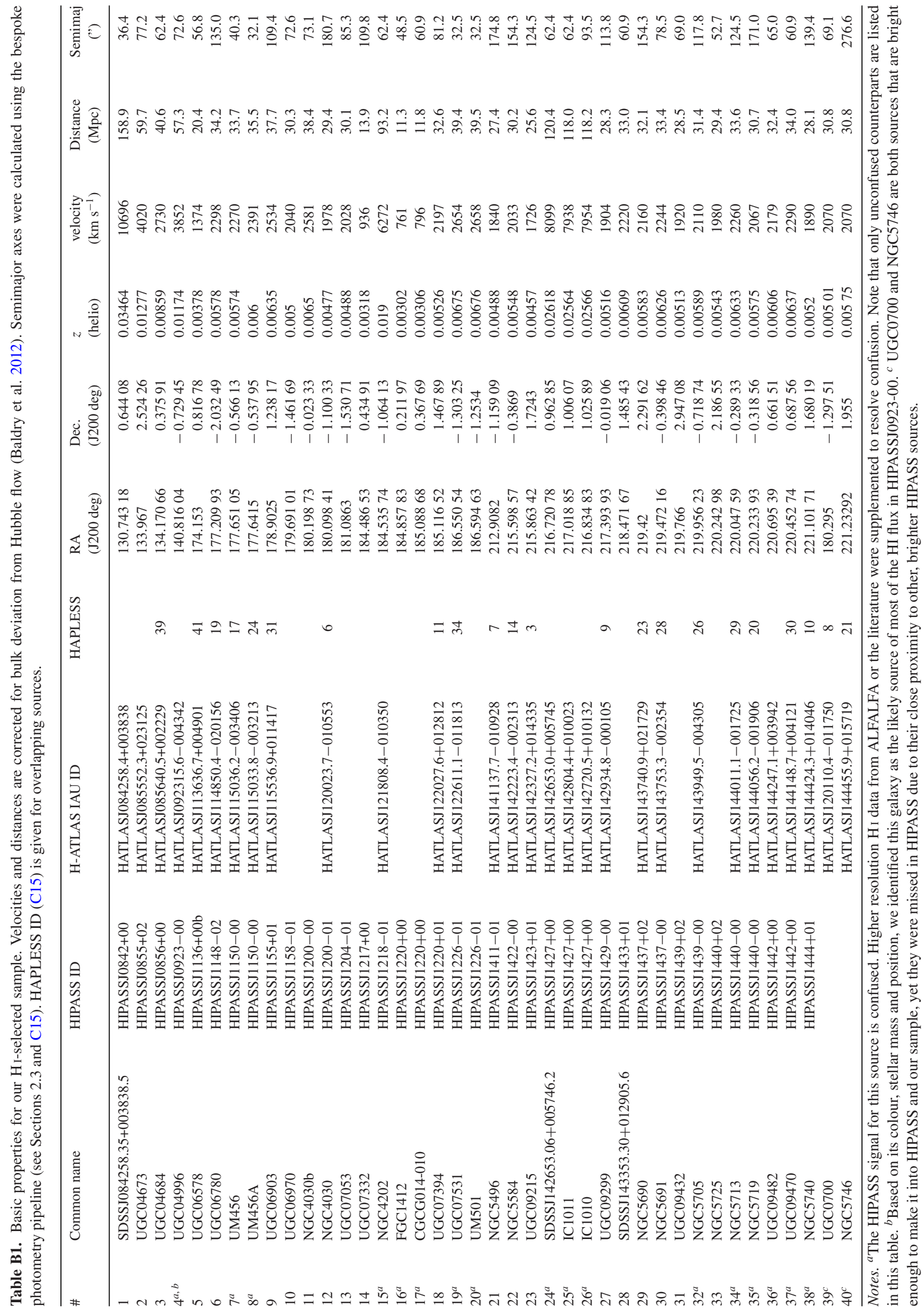




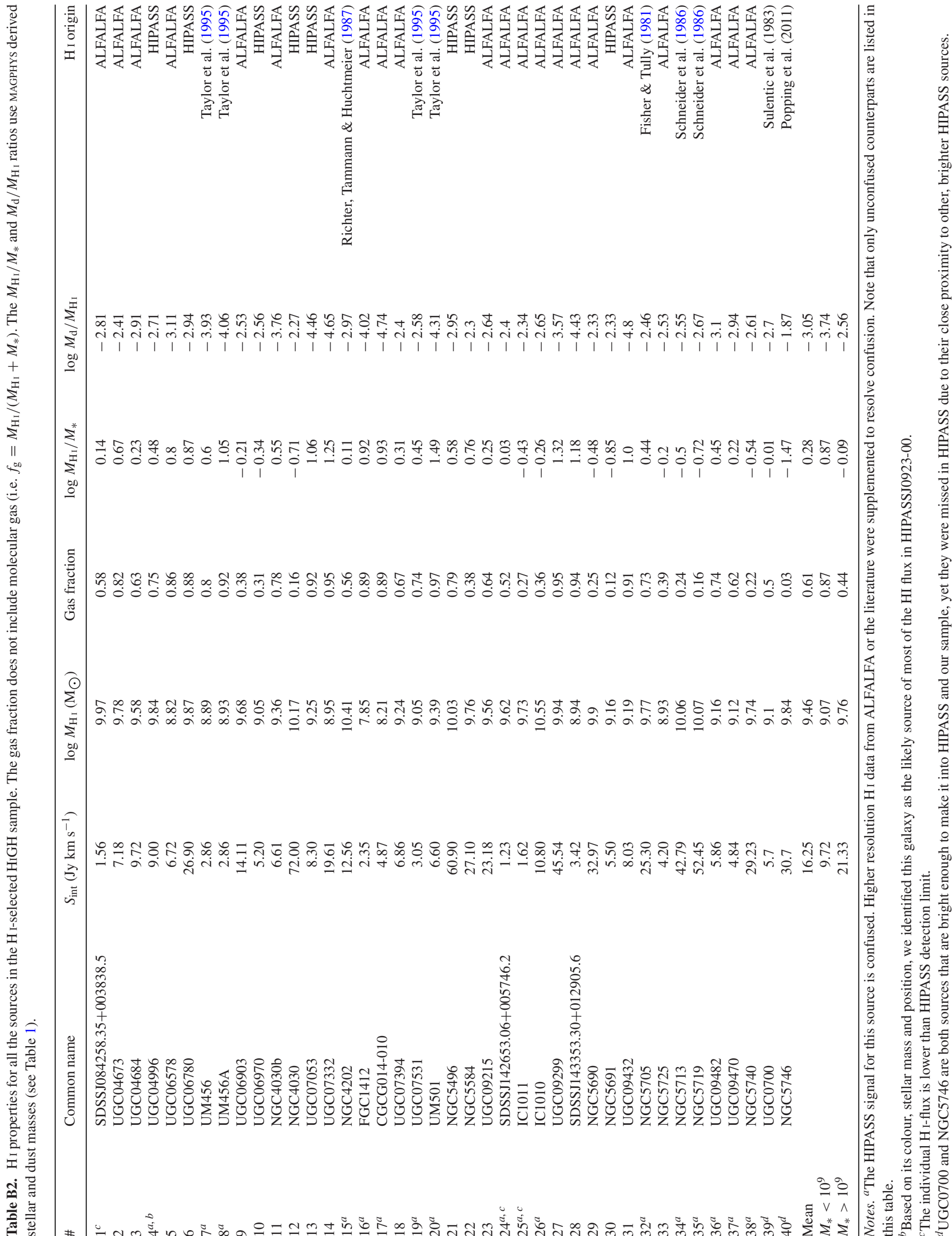




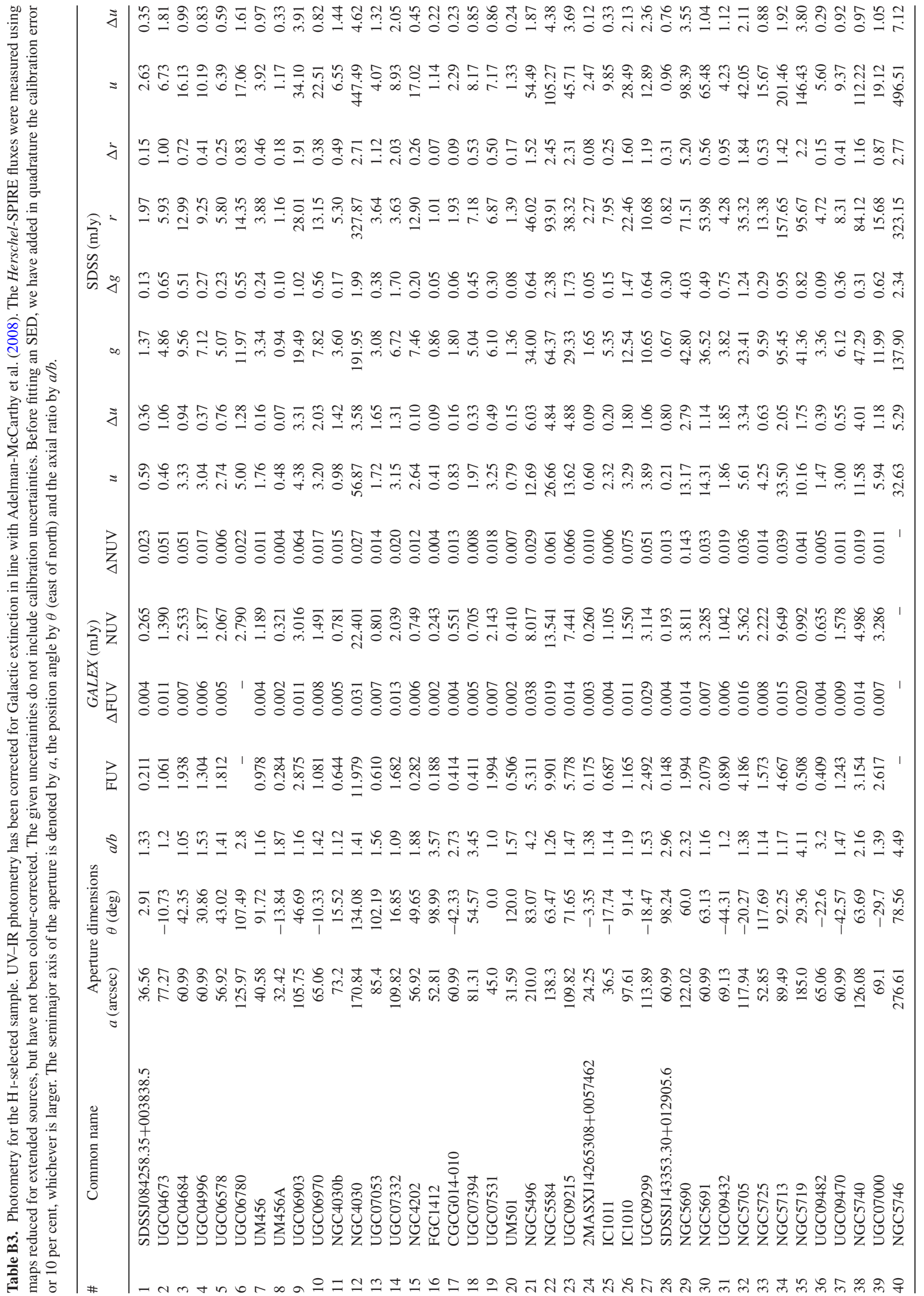




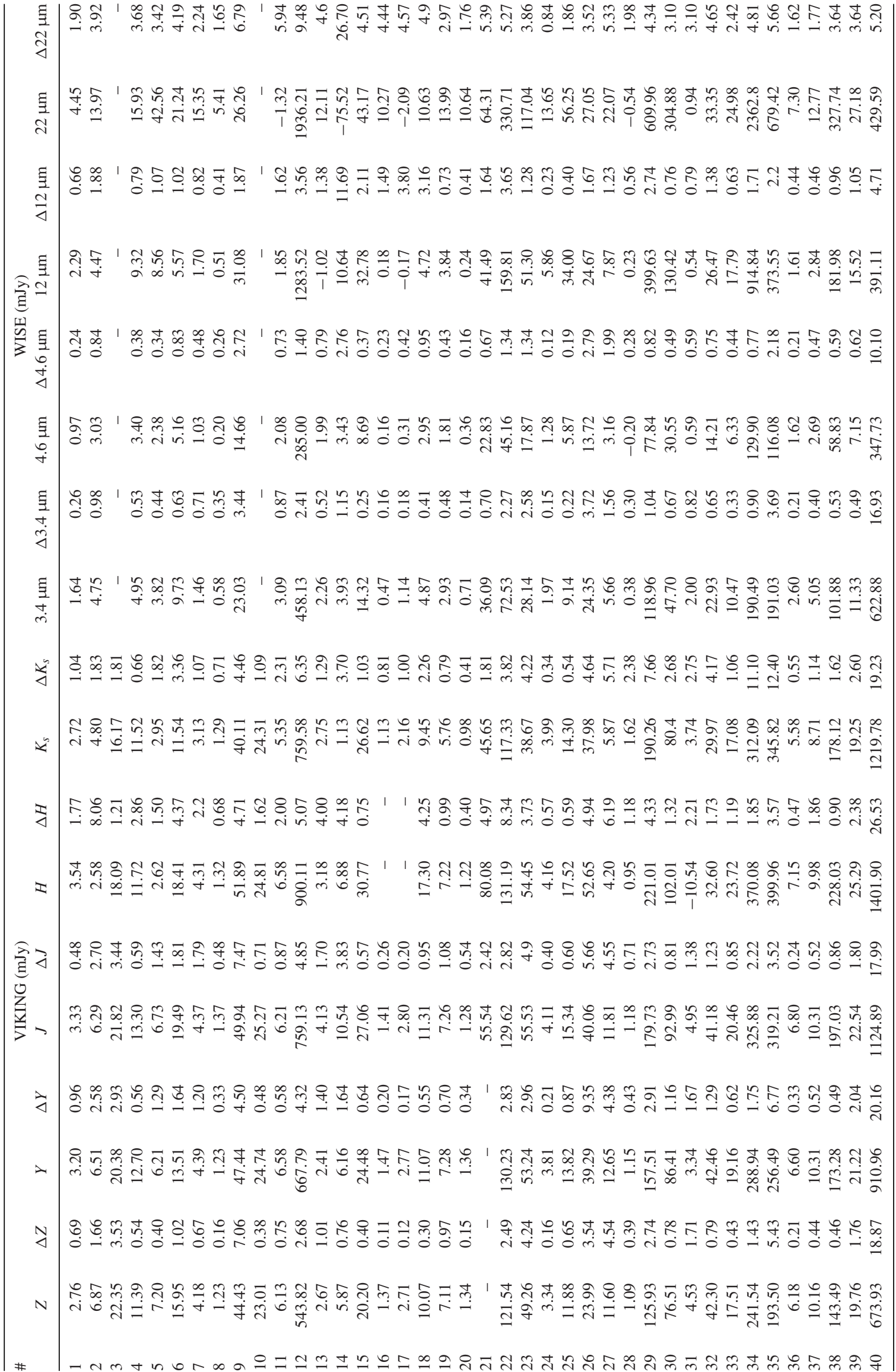




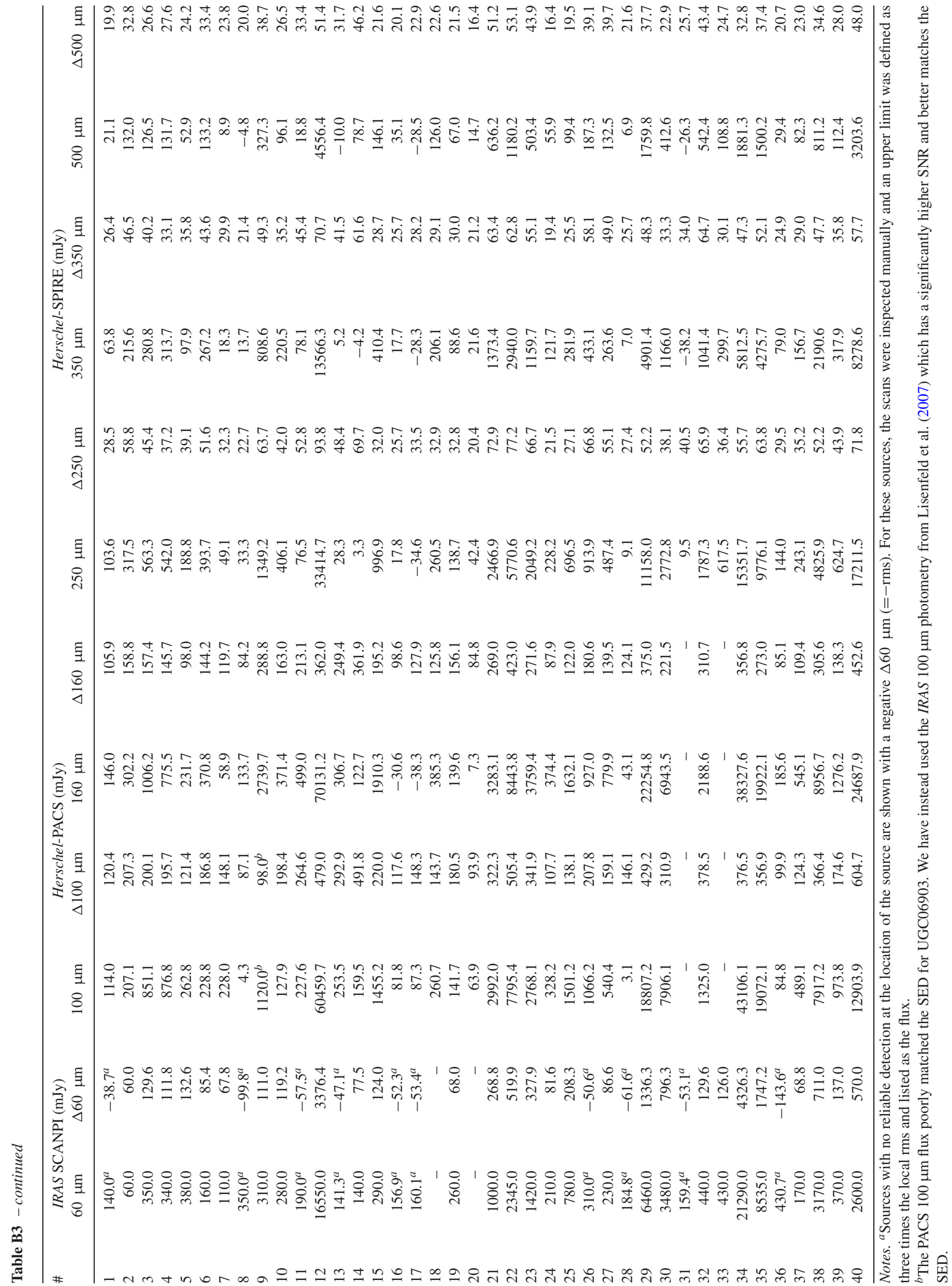



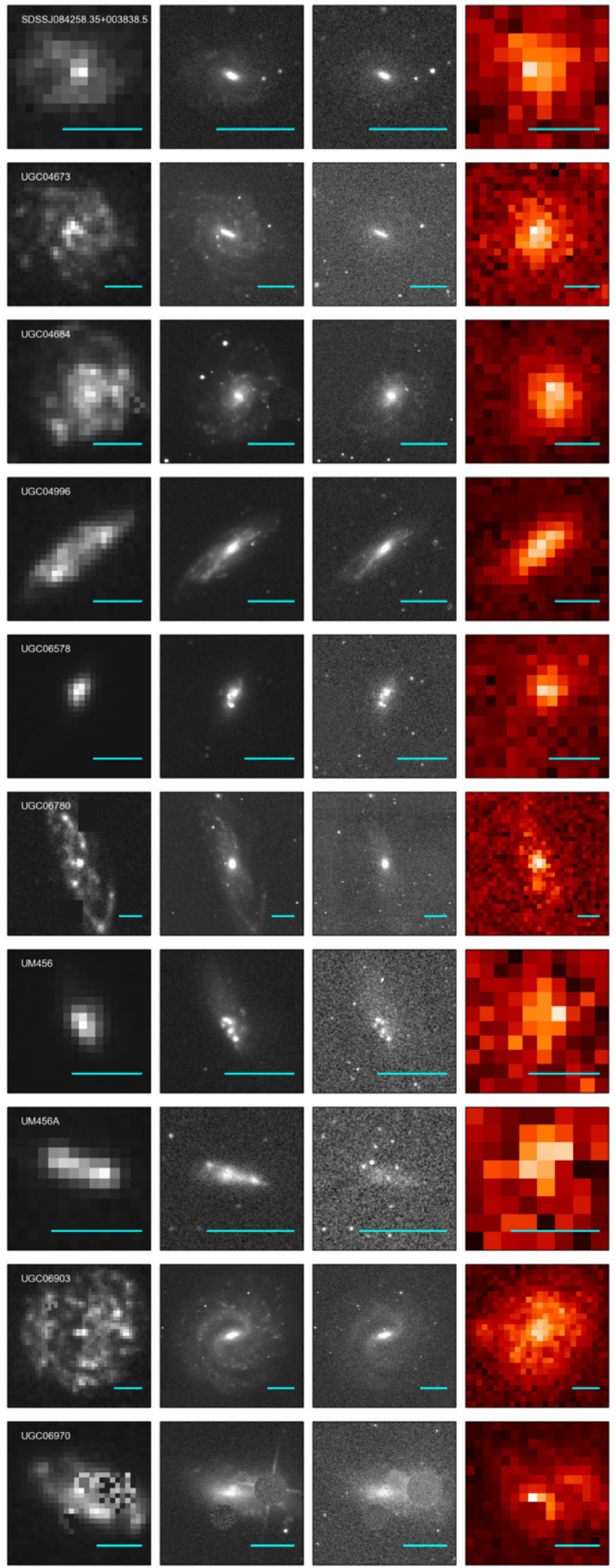
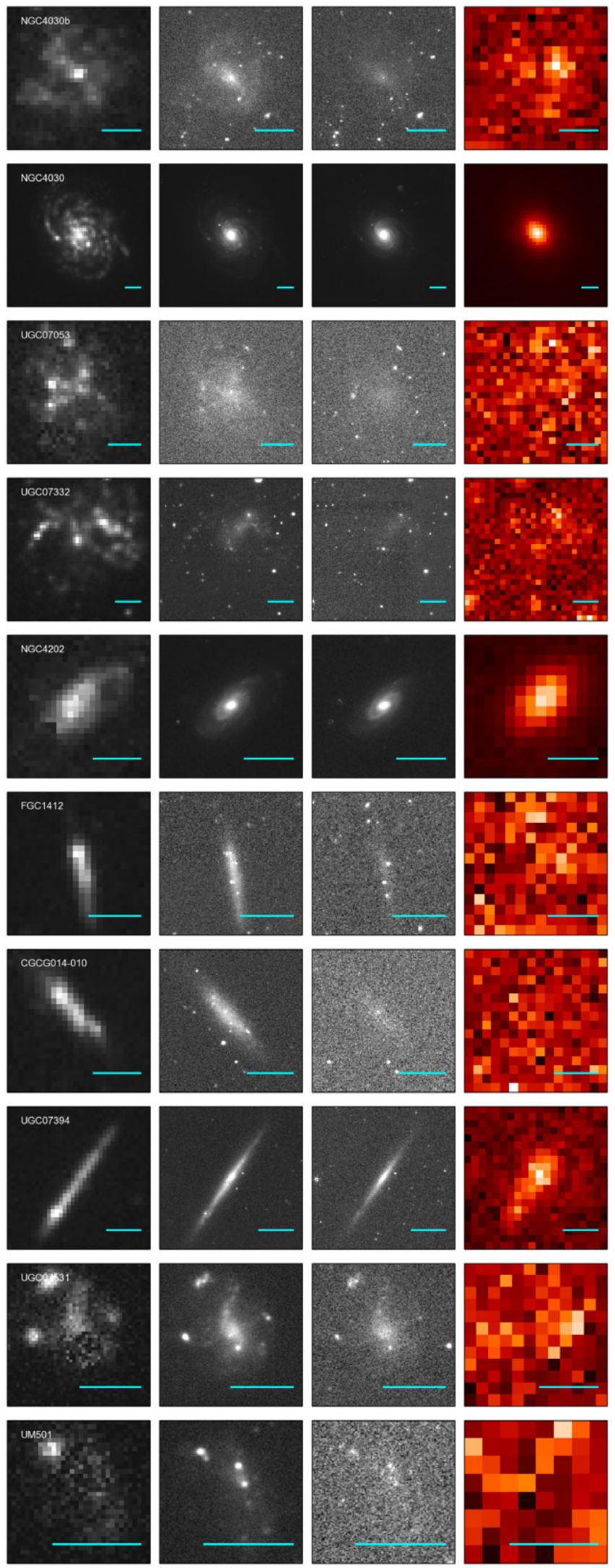

Figure B1. Multiwavelength images for all the $\mathrm{H}$ I-selected sources. The brightest foreground stars and background galaxies have been subtracted and replaced by adjacent pixels. The bands displayed, from left-to-right, are: GALEX FUV, SDSS $r$ band, VIKING $K_{\mathrm{S}}$ band and Herschel $250 \mu \mathrm{m}$. The size of each cut-out is 1.5 times the semimajor axis of the aperture and a scale bar with a length of 30 arcsec is shown on each image in cyan. 

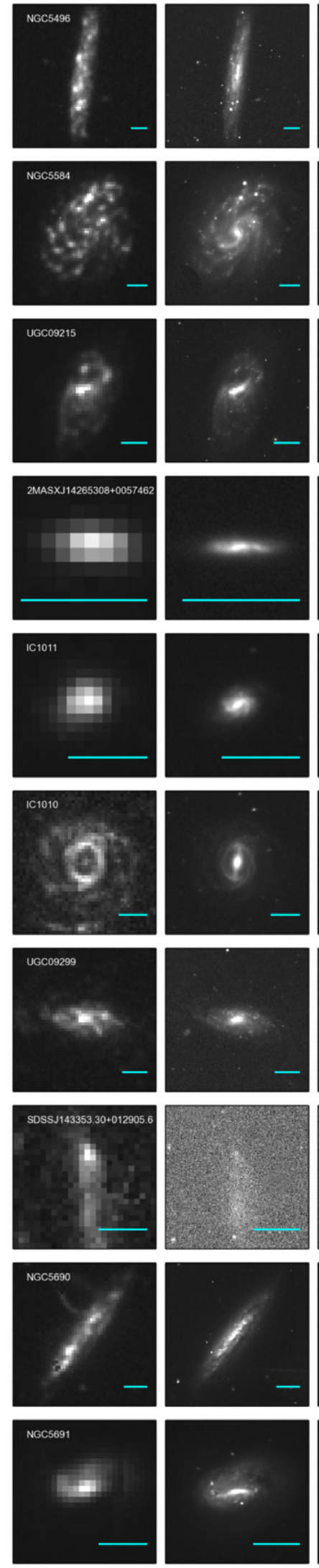
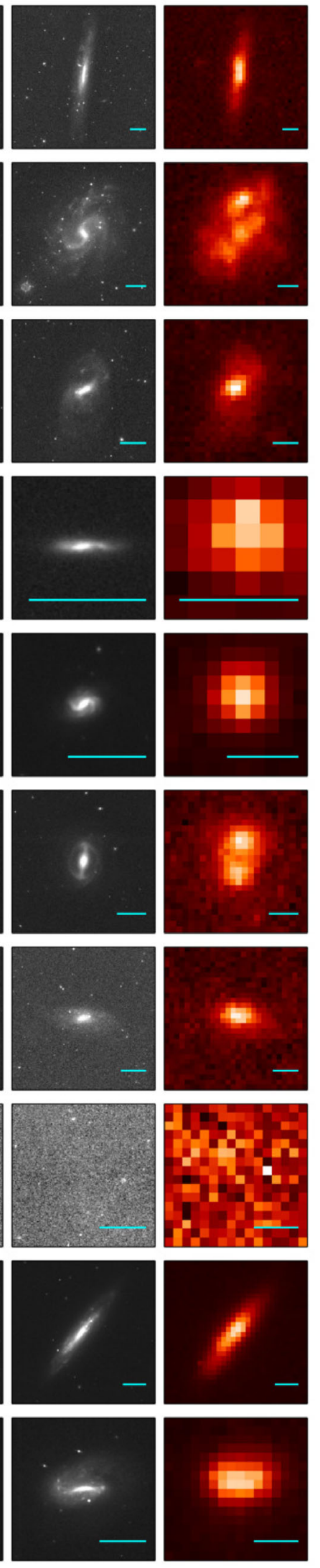
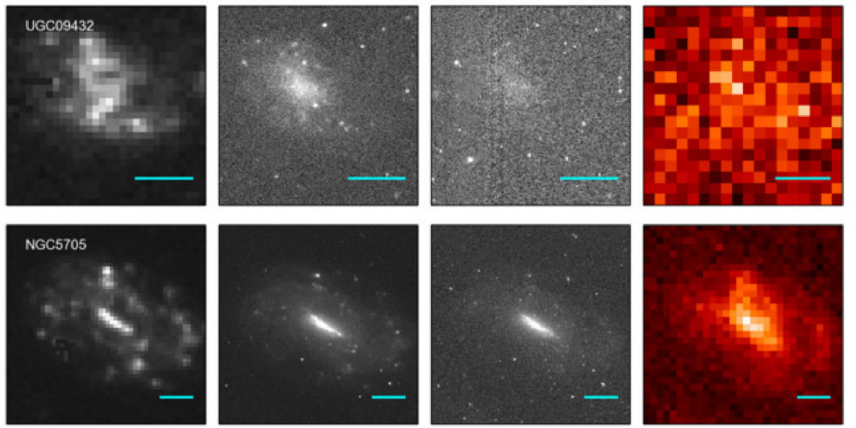
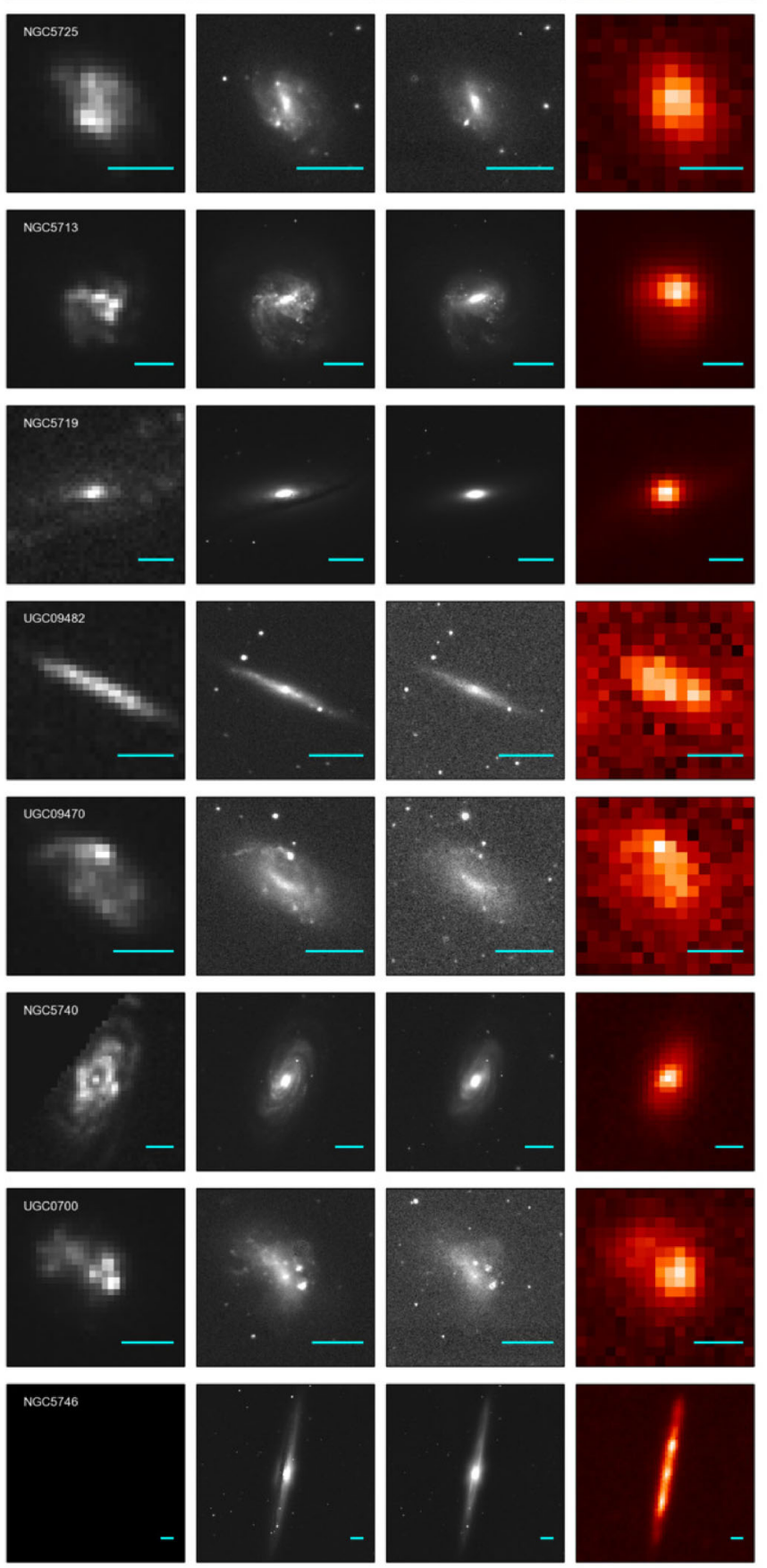

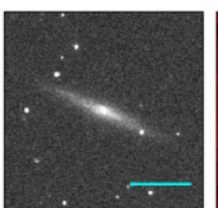

Figure B1 - continued 

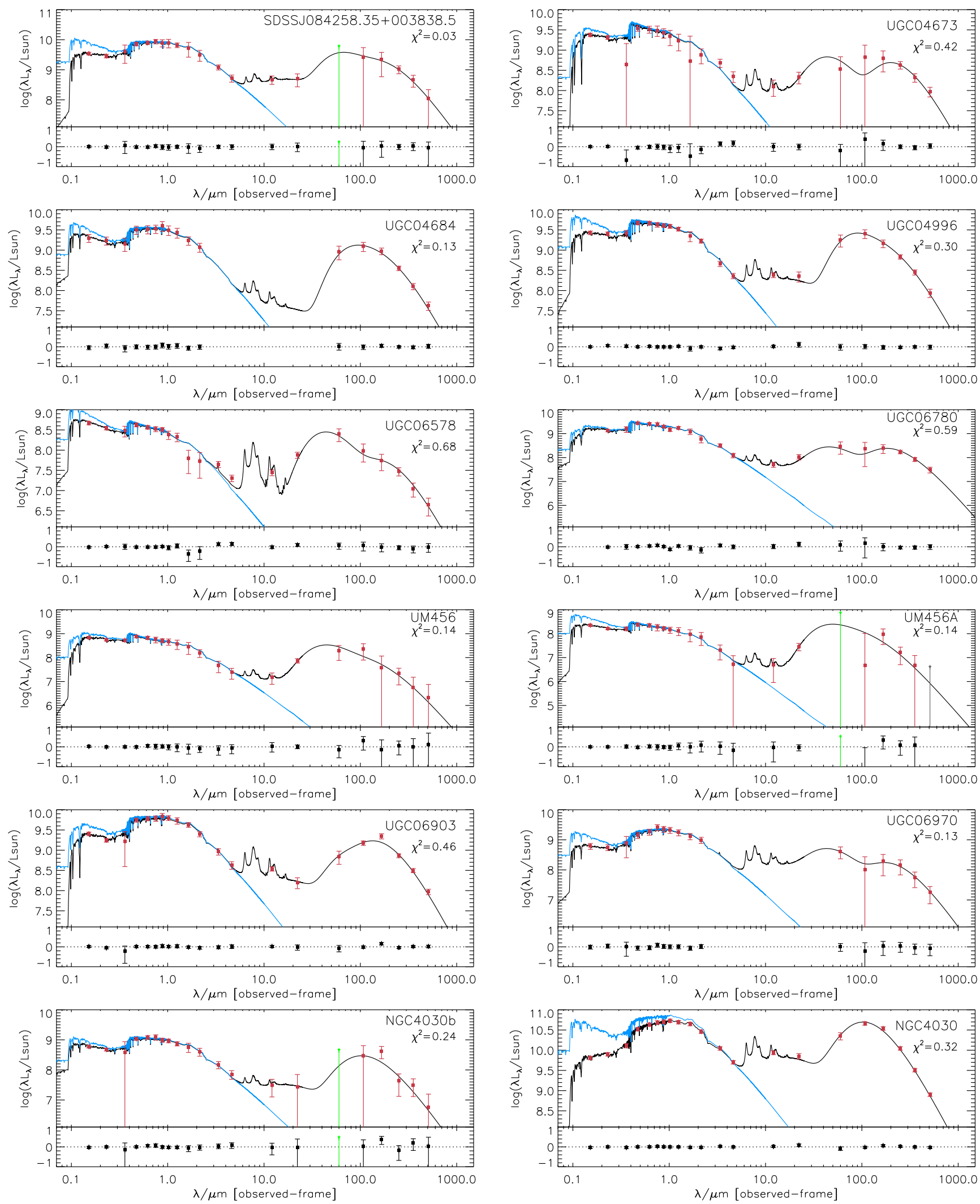

Figure B2. Multiwavelength SEDs of the $40 \mathrm{H}$ I-selected sources in HIGH, with observed photometry (red points) from FUV to the submm. The photometry process (including determination of errors) is described in Section 2.3. IRAS60 $3 \sigma$ upper limits are shown as green triangles. Since negative fluxes cannot be plotted on a logarithmic scale, we have plotted the $1 \sigma$ upper limits as orange triangles. The solid black line is the best-fitting model SED and the solid blue line is the unattenuated optical model. The residuals of the fit are shown in the panel below each SED. The shown $\chi^{2}$ 's are the total $\chi^{2}$ divided by the number of bands, as given by the standard version of MAGPHYs. 

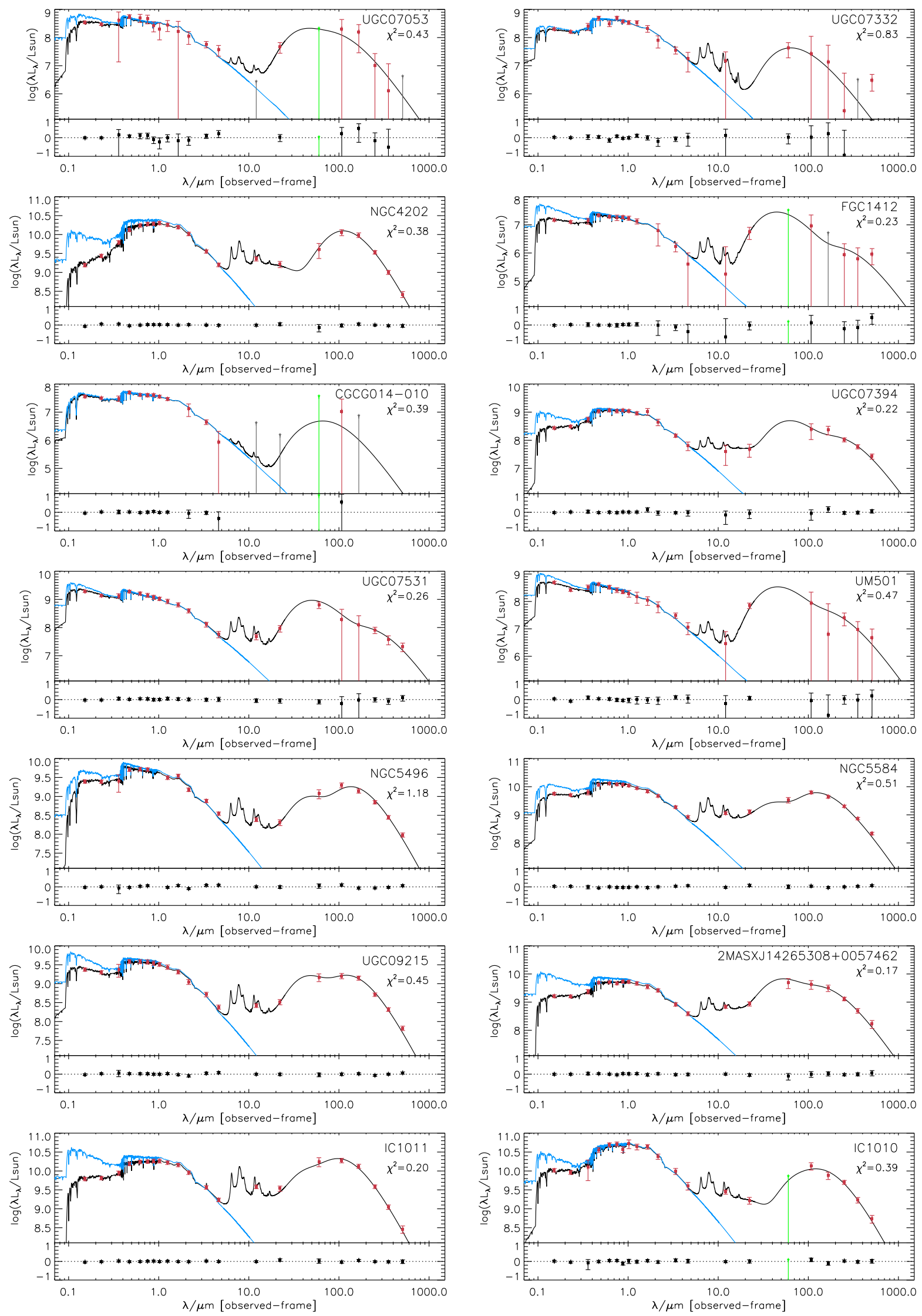

Figure B2 - continued 

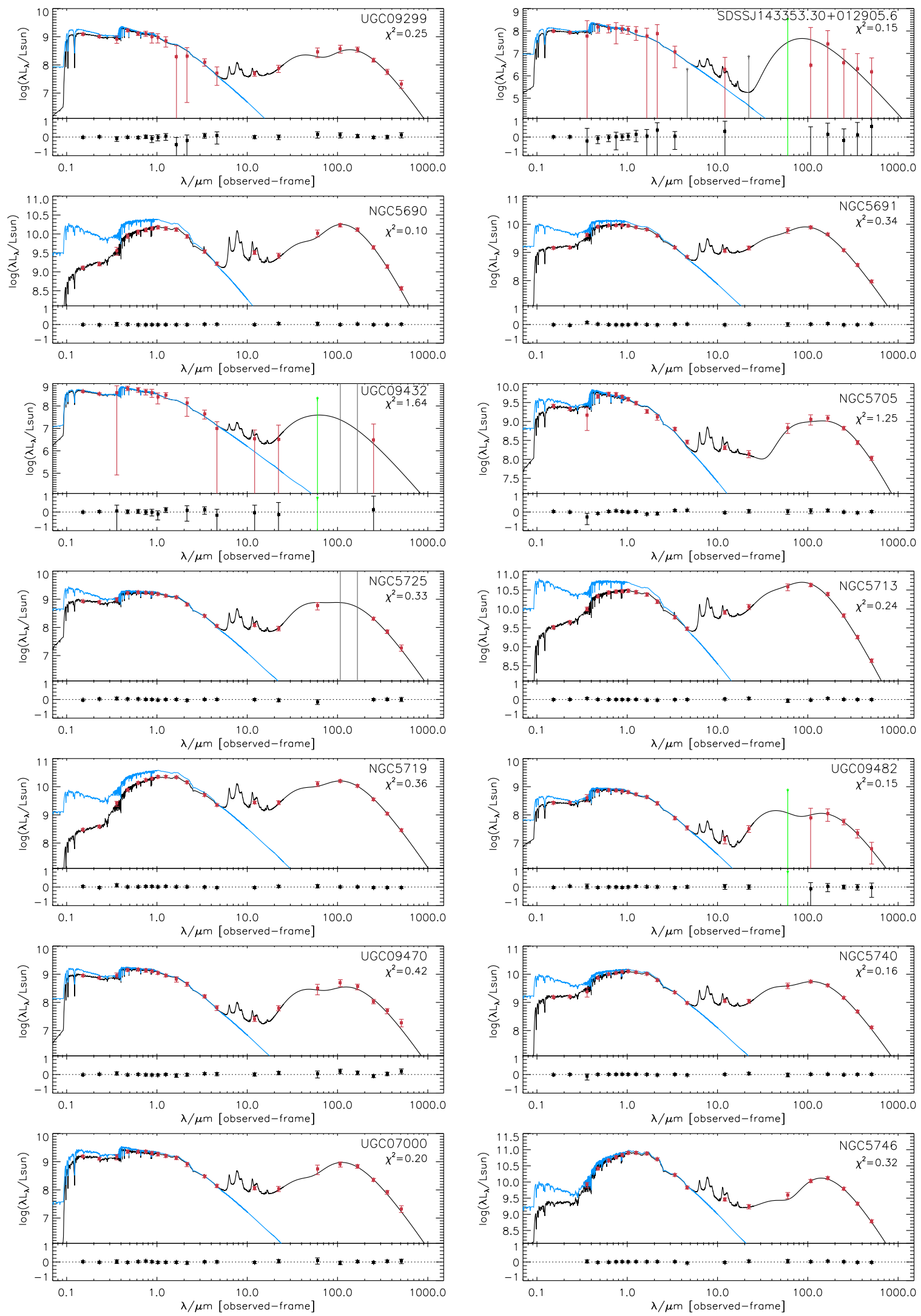

Figure B2 - continued

This paper has been typeset from a $\mathrm{T}_{\mathrm{E}} \mathrm{X} / \mathrm{L} \mathrm{T} \mathrm{E} \mathrm{X}$ file prepared by the author. 